\title{
Strichartz Estimates for Some 2D Water Wave Models
}

\author{
José R. Quintero ${ }^{1, *}$ and Alex M. Montes ${ }^{2}$ \\ ${ }^{1}$ Departamento Matemáticas, Universidad del Valle, A. A. 25360 Cali, Colombia \\ 2 Departamento Matemáticas, Universidad del Cauca, Carrera 3 No 3N-100 Popayán, Colombia
}

Received: 14 Mar. 2013, Revised: 17 Jul. 2013, Accepted: 18 Jul. 2013

Published online: 1 Nov. 2013

\begin{abstract}
In this paper we establish Strichartz type estimates associated with a class of semigroup operators in $\mathbb{R}^{n}$, which for $n=2$ correspond to some 2D water wave models. We also establish a nonlinear scattering result for solutions of the generalized Benney-Luke equation for higher order nonlinearity and small data initial in the energy space.
\end{abstract}

Keywords: Strichartz estimates, water waves models, nonlinear scattering.

\section{Introduction}

The formulation of some phenomena in nonlinear optics, fluid dynamics, nuclear physics, plasma physics, biosciences and several other areas can be reduced in some cases to studying nonlinear evolution equations in mathematical physics and applied mathematics, among other. For these nonlinear evolution models, it is very important to determine existence and uniqueness of solution, and the existence of special solutions as travelling waves solutions (periodic and solitary waves). For instance, solitary waves are important in the study of dynamics of wave propagation in many applied models such as fluid dynamics, optics, acoustics, oceanography, and weather forecasting. As it is well known now, an important application is the use of optical solitons (solitary waves of finite energy) in fibers as an efficient (reliable and fast) means of long-distance communication ([1], [4], [6], [7], [13], [14], [15], [18], [23]).

Since the works by I. Segal ([21]) and R. Strichartz ([24]) related with the study of the Cauchy problem for some nonlinear wave models and diverse space-time estimates for solutions of homogeneous and nonhomogeneous problems, there has been a huge number of papers using Strichartz type estimates for the study of different PDE's in the framework of some function spaces (Sobolev spaces, Besov space, $L^{p}$ spaces, distributional spaces, for example) to establish well-posedness and scattering properties.

In this paper we are interested in obtaining Strichartz type estimates in $\mathbb{R}^{n}$ associated with some special semigroups which are related with water waves models for $n=2$, as the wave equation, the generalized Benney-Luke equation and some Boussinesq type models (see [13], [15]). In particular, for the generalized Benney-Luke model with a nonlinearity of order $m$ large enough, we establish nonlinear scattering for small data in the energy space to get the asymptotic behavior of global solutions as $t \rightarrow \pm \infty$.

Models considered in this paper are related with the evolution of long water waves with small amplitude of an irrotational, three-dimensional flow of an inviscid, incompressible fluid which at rest occupies the region $(x, y) \in \mathbb{R}^{2}$ and $0<z<h_{0}$. It is known that this type of models can be described as an approximation of the full water wave problem imposing some restrictions on the parameters that affect the propagation of gravity water waves as the nonlinearity (amplitude parameter $\varepsilon$ ), the dispersion (long-wave parameter $\mu$ ) and the horizontal spreading. In these models, the variable $\eta(x, y, t)$ represents the free surface elevation and the variable $\Phi$ represents essentially the velocity potential at the bottom, after the approximation process using Taylor expansion on the variable $z$ (see [2], [3], [9], [10], [11], [13], [14], [15], [16]).

This paper is organized as follows. In section 2, we include some preliminaries. In section 3, we establish Strichartz estimates for a general class of semigroups defined in $\mathbb{R}^{n}$. As for many models, the Strichartz estimates are obtained by combining the Littlewood-Paley dyadic decomposition, the Riesz-Thorin Interpolation Theorem, the Hardy-Littlewood-Sobolev Inequality and

\footnotetext{
*Corresponding author e-mail: jose.quintero@ correounivalle.edu.co
} 
duality arguments. In section 4, we verify the hypotheses to have the Strichartz estimates for the semigroup associated with some water wave models as the wave equation, the generalized Benney-Luke equation and some Boussinesq-Benney-Luke type systems. In section 5 , we prove a nonlinear scattering result for the generalized Benney-Luke equation and analyze the asymptotic behavior of global solutions for this model as $t \rightarrow \pm \infty$.

\section{Preliminaries.}

We will use the following notation, $L^{p}\left(\mathbb{R}^{n}\right), 1 \leq p \leq \infty$, denote the usual Lebesgue space with the norma $\|\cdot\|_{p}=\|\cdot\|_{L^{p}\left(\mathbb{R}^{n}\right)}$. The usual Sobolev space $H^{s, p}\left(\mathbb{R}^{n}\right), s \in \mathbb{R}$ is defined as the closure of Schwartz space $\mathcal{S}\left(\mathbb{R}^{n}\right)$ with respect to the norm given by

$$
\|f\|_{H^{s, p}\left(\mathbb{R}^{n}\right)}=\left\|\mathcal{F}^{-1}\left(\left(1+|\xi|^{2}\right)^{\frac{s}{2}} \widehat{f}\right)\right\|_{L^{p}\left(\mathbb{R}^{n}\right)}
$$

where the Fourier transform of a function $w$ defined on $\mathbb{R}^{n}$ is given by

$$
(\mathcal{F} w)(\xi)=\widehat{w}(\xi)=\frac{1}{(2 \pi)^{\frac{n}{2}}} \int_{\mathbb{R}^{n}} e^{-i x \cdot \xi} w(x) d x .
$$

The homogeneous Sobolev space $\dot{H}^{s, p}\left(\mathbb{R}^{n}\right)$ is defined as the closure of Schwartz space $\mathcal{S}\left(\mathbb{R}^{n}\right)$ with respect to the norm

$$
\|f\|_{\dot{H}^{s, p}\left(\mathbb{R}^{n}\right)}=\left\|\mathcal{F}^{-1}\left(|\xi|^{s} \widehat{f}\right)\right\|_{L^{p}\left(\mathbb{R}^{n}\right)} .
$$

Especially we write $H^{s}\left(\mathbb{R}^{n}\right)=H^{s, 2}\left(\mathbb{R}^{n}\right)$ and $\dot{H}^{s}\left(\mathbb{R}^{n}\right)=\dot{H}^{s, 2}\left(\mathbb{R}^{n}\right)$. The space $\mathcal{V}^{s}\left(\mathbb{R}^{n}\right)$ denote the closure of $\mathcal{S}\left(\mathbb{R}^{2}\right)$ with respect to the norm given by

$$
\|f\|_{\mathcal{V}^{s}\left(\mathbb{R}^{n}\right)}=\|\nabla f\|_{H^{s-1}\left(\mathbb{R}^{n}\right)}=\||\xi| \widehat{f}\|_{H^{s-1}\left(\mathbb{R}^{n}\right)} .
$$

We also adopt the notation

$$
\|f\|_{L_{t}^{q}\left(L_{x}^{p}\right)}=\left(\int_{-\infty}^{\infty}\|f(\cdot, t)\|_{L^{p}\left(\mathbb{R}^{n}\right)}^{q} d t\right)^{1 / q}
$$

with the obvious modification when $q=\infty$. Hereafter $C$ denotes a generic constant whose value may change from instance to instance. The following lemma will be frequently used.

Lemma 1. Let $s, r \in \mathbb{R}$ and $1 \leq p<\infty$. Then we have that 1) If $s \geq r+n\left(\frac{1}{p}-\frac{1}{q}\right)$ and $1<p \leq q<\infty$, then $H^{s, p}\left(\mathbb{R}^{n}\right) \hookrightarrow$ $H^{r, q}\left(\mathbb{R}^{n}\right)$.

2) If $s>\frac{n}{p}$, then $H^{s, p}\left(\mathbb{R}^{n}\right) \hookrightarrow L^{\infty}\left(\mathbb{R}^{n}\right)$.

\subsection{Littlewood-Paley decomposition}

The main tool used to obtain Strichartz estimates for many models is the Littlewood-Paley dyadic decomposition, which are defined in the following standard way. Let $\chi \in C_{0}^{\infty}\left(\mathbb{R}^{n}\right)$ be a radial cut-off function such that $0 \leq \chi \leq 1$ and

$$
\chi(\xi)= \begin{cases}1 & \text { if }|\xi| \leq 1 / 2 \\ 0 & \text { if }|\xi| \geq 1\end{cases}
$$

If $\beta(\xi)=\chi(\xi)-\chi(2 \xi)$, then $\beta \in C_{0}^{\infty}\left(\mathbb{R}^{n}\right)$ is a radial function supported away from zero such that for any $\xi \in \mathbb{R}^{2} \backslash\{0\}$,

$$
\sum_{j=-\infty}^{\infty} \beta\left(\frac{\xi}{2^{j}}\right)=1
$$

Now, define the operator $\Delta_{j}: \mathcal{S}\left(\mathbb{R}^{n}\right) \rightarrow \mathcal{S}\left(\mathbb{R}^{n}\right)$ by

$$
\widehat{\Delta_{j} f}(\xi)=\beta\left(\frac{\xi}{2^{j}}\right) \widehat{f}(\xi), \text { for } j \in \mathbb{Z} .
$$

Then $f=\sum_{j=-\infty}^{\infty} \Delta_{j} f$ in $\mathcal{S}\left(\mathbb{R}^{2}\right)$. Moreover, we see that

$$
\operatorname{supp} \widehat{\Delta_{j} f} \subset\left\{2^{j-2} \leq|\xi| \leq 2^{j}\right\}
$$

and also that if $|j-k| \geq 2$ then

$$
\left(\Delta_{j} f\right)\left(\Delta_{k} f\right)=0 \text {. }
$$

We remember that $\left\{\Delta_{j} f\right\}_{j}$ is called the Littlewood-Paley decomposition of $f$. We will use the coming important result.

Lemma 2. Let $s \in \mathbb{R}$, then holds the following statements. 1) Given $2 \leq p<\infty$, there exist $C=C_{p, n, s}>0$ such that

$$
\begin{aligned}
& \|f\|_{H^{s, p}\left(\mathbb{R}^{n}\right)}^{2} \leq C \sum_{j=-\infty}^{\infty}\left\|\Delta_{j} f\right\|_{H^{s, p}\left(\mathbb{R}^{n}\right)}^{2}, \\
& \|f\|_{\dot{H}^{s, p}\left(\mathbb{R}^{n}\right)}^{2} \leq C \sum_{j=-\infty}^{\infty}\left\|\Delta_{j} f\right\|_{\dot{H}^{s, p}\left(\mathbb{R}^{n}\right)}^{2} .
\end{aligned}
$$

2) Given $1<p \leq 2$, there exist $C=C_{p, n, s}>0$ such that

$$
\begin{aligned}
& \sum_{j=-\infty}^{\infty}\left\|\Delta_{j} f\right\|_{H^{s, p}\left(\mathbb{R}^{n}\right)}^{2} \leq C\|f\|_{H^{s, p}\left(\mathbb{R}^{n}\right)}^{2}, \\
& \sum_{j=-\infty}^{\infty}\left\|\Delta_{j} f\right\|_{\dot{H}^{s, p}\left(\mathbb{R}^{n}\right)}^{2} \leq C\|f\|_{\dot{H}^{s, p}\left(\mathbb{R}^{n}\right)}^{2} .
\end{aligned}
$$

Also, we will use the following version of Bernstein's Lemma.

Lemma 3. [Bernstein's Lemma] Assume $f \in L^{p}\left(\mathbb{R}^{n}\right), 1 \leq$ $p \leq \infty$ such that

$$
\text { supp } \widehat{f} \subset\left\{\xi \in \mathbb{R}^{n}: 2^{j-2} \leq|\xi| \leq 2^{j}\right\} .
$$

Then for every $s \geq 0$ there exist $C=C_{p, n, s}>0$ such that

$$
\|f\|_{\dot{H}^{s, p}\left(\mathbb{R}^{n}\right)} \leq C 2^{j s}\|f\|_{L^{p}\left(\mathbb{R}^{n}\right)} .
$$




\section{Strichartz estimates for a class of semigroups}

In this section we establish Strichartz type estimates in the case of semigroup $S(t)$ that are linear combination of operators $S_{k}(t)$ of the form

$$
\widehat{S_{k}(t) f}(\xi)=\Lambda_{k}(\xi) e^{i t|\xi| \rho(|\xi|)} \widehat{f}(\xi), \quad \xi \in \mathbb{R}^{n}
$$

where $1 \leq k \leq 3$ and

$$
\Lambda_{1}(\xi)=\varphi_{1}(\xi), \Lambda_{2}(\xi)=|\xi| \varphi_{2}(\xi), \Lambda_{3}(\xi)=\frac{\varphi_{3}(\xi)}{|\xi|}
$$

with

$$
\varphi_{k} \in L^{\infty}\left(\mathbb{R}^{n}\right)
$$

If $\rho= \pm 1$ and $\Lambda_{1}=1$, we have that semigroup $S_{1}^{ \pm}(t)$ is associated with the wave equation in $\mathbb{R}^{n}$. We will see in section 4 that the semigroup associated with some twodimensional water wave type models have exactly the form $S_{k}$ defined for (3) in $\mathbb{R}^{2}$.

We are interested in deriving some Strichartz estimates for general semigroups, as possible. So, hereafter we will assume that $\rho: \mathbb{R} \rightarrow \mathbb{R}$ is a $C^{n}(\mathbb{R})$ function. For $j \in \mathbb{Z}$, we define functions $\rho_{j}$ and $h_{j}$ by

$$
\rho_{j}(r)=\rho\left(2^{j} r\right), \quad h_{j}(r)=\rho_{j}(r)+r \rho_{j}^{\prime}(r) .
$$

We will assume that the function $\rho$ satisfies the condition:

[H] Given the interval $\left[r_{1}, r_{2}\right] \subset \mathbb{R}^{+}$, there is $c_{l}>0$ such that for all $(r, j) \in\left[r_{1}, r_{2}\right] \times \mathbb{Z}$,

$$
\left|h_{j}(r)\right| \geq c_{0}, \quad \frac{\left|h_{j}^{(l)}(r)\right|}{\left|h_{j}(r)\right|} \leq c_{l}, \quad 1 \leq l \leq n-1 .
$$

Note that for the wave equation, we have that $\rho= \pm 1$, meaning that the hypothesis $[\mathbf{H}]$ is trivial. We will see that conditions on $\rho$ and $\varphi_{k}$ are verified explicitly for some Boussinesq type models in section 4 for $n=2$, which corresponds to the physical problem. The technical condition $[\mathbf{H}]$ on the function $\rho$ appears when we try to truncate the semigroup Fourier symbol $\widehat{S_{k}(t)}$ appropriately, as done for the water wave equation.

\subsection{Local estimates}

We obtain some Strichartz estimates by performing a slight modification of the Strichartz estimates used for wave equation (see [8], [20], [24]). As in the mention works, we use a very well known result related with the pointwise estimate for the Fourier transform restricted to the $(n-1)$-sphere (see T. Wolff, [25]), in order to obtain estimates for some operators.
Lemma 4. Let $n \geq 2$ and $S^{n-1}$ the unit sphere in $\mathbb{R}^{n}$. Define

$$
\check{\sigma}_{n}(x)=\int_{S^{n-1}} e^{i x \cdot \xi} d S_{\xi}, \quad x \in \mathbb{R}^{n},
$$

then we have that

$$
\left|\check{\sigma}_{n}(x)\right| \leq C|x|^{-\frac{n-1}{2}} .
$$

In order to define the truncated operator, we choose a radial cutoff function $\beta \in C_{0}^{+\infty}\left(\mathbb{R}^{n}\right)$ supported away from zero. So, if $j \in \mathbb{Z}$ define the operator $T_{j}$, for $(x, t) \in \mathbb{R}^{n+1}$, as

$$
\left(T_{j}(t) f\right)(x)=\frac{1}{(2 \pi)^{\frac{n}{2}}} \int_{\mathbb{R}^{n}} e^{i x \cdot \xi} e^{i t|\xi| \rho\left(2^{j}|\xi|\right)} \beta(\xi) \widehat{f}(\xi) d \xi
$$

Note that

$$
\left(T_{j}(t) f\right)(x)=\left(K_{j}(t) * f\right)(x)
$$

where

$$
\left(K_{j}(t)\right)(x)=\frac{1}{(2 \pi)^{\frac{n}{2}}} \int_{\mathbb{R}^{n}} e^{i x \cdot \xi} e^{i t|\xi| \rho\left(2^{j}|\xi|\right)} \beta(\xi) d \xi .
$$

The following results describes some estimates of the family of operators $\left\{T_{j}\right\}_{j \in \mathbb{Z}}$ on $L^{p}\left(\mathbb{R}^{n}\right)$.

Lemma 5. There exist $C=C_{n}>0$ (not depending on $j$ ) such that

$$
\left\|T_{j}(t) f\right\|_{2} \leq C\|f\|_{2}
$$

Moreover, if $n \geq 2$ and $\rho$ satisfies $[\mathbf{H}]$, then

$$
\left\|T_{j}(t) f\right\|_{\infty} \leq C|t|^{\frac{-(n-1)}{2}}\|f\|_{1} .
$$

Proof. Let $g(\xi)=e^{i t|\xi| \rho\left(2^{j}|\xi|\right)} \beta(\xi)$. Using Plancherel Theorem we have that

$$
\left\|T_{j}(t) f\right\|_{2}=\|g \widehat{f}\|_{2} \leq C\|\widehat{f}\|_{2}=C\|f\|_{2}
$$

as claimed in the first inequality. Now we will show the second estimate. First assume that $|t| \leq 1$. Then using Young's inequality we find that

$$
\left\|T_{j}(t) f\right\|_{\infty} \leq\left\|K_{j}(t)\right\|_{\infty}\|f\|_{1} \leq C\|f\|_{1} \leq C|t|^{\frac{-(n-1)}{2}}\|f\|_{1} .
$$

Now, we consider the case $|t|>1$. We will prove that there exist $C>0$ such that for all $j \in \mathbb{Z}$,

$$
\left\|K_{j}(t)\right\|_{\infty} \leq C|t|^{\frac{-(n-1)}{2}} .
$$

Recall that $\beta$ is assumed to be radial and supported away from zero. Using polar coordinates $\xi=r \omega$ we have that

$$
\begin{aligned}
(2 \pi)^{\frac{n}{2}}\left(K_{j}(t)\right)(x) & =\int_{0}^{\infty} \int_{S^{n-1}} e^{i r\left(x \cdot \omega+t \rho_{j}(r)\right)} a(r) d S_{\omega} d r \\
& =\int_{0}^{\infty} \check{\sigma}_{n}(r x) e^{i t r \rho_{j}(r)} a(r) d r
\end{aligned}
$$


where $a$ is a smooth function such that

$$
\text { suppa } \subset\left\{r \in \mathbb{R}: 0<r_{1} \leq|r| \leq r_{2}\right\} .
$$

Now, let $c_{0}>0$ such that $\left|h_{j}(r)\right| \geq c_{0}$ for all $j \in \mathbb{Z}$ (see (5)). We consider two cases.

Case 1. Suppose $c_{0}|t|<2|x|$. Using estimate (6) in Lemma 4 , we have that

$$
\begin{aligned}
\left|\left(K_{j}(t)\right)(x)\right| & \leq C \int_{0}^{\infty}\left|\check{\sigma}_{n}(r x)\right||a(r)| d r \\
& \leq C \int_{0}^{\infty}|r x|^{\frac{-(n-1)}{2}}|a(r)| d r \\
& \leq C|t|^{\frac{-(n-1)}{2}} .
\end{aligned}
$$

Case 2. Suppose $c_{0}|t| \geq 2|x|$. From the integration by parts formula we see that

$$
\begin{aligned}
I & =\int_{0}^{\infty} e^{i r\left(x \cdot \omega+t \rho_{j}(r)\right)} a(r) d r \\
& =\int_{0}^{\infty} \frac{d}{d r}\left(e^{i r\left(x \cdot \omega+t \rho_{j}(r)\right)}\right) \frac{a(r)}{i\left(x \cdot \omega+t h_{j}(r)\right)} d r \\
& =\int_{0}^{\infty} e^{i r\left(x \cdot \omega+t \rho_{j}(r)\right)} F(r) d r,
\end{aligned}
$$

where

$$
F(r)=\frac{-a^{\prime}(r)}{i\left(x \cdot \omega+t h_{j}(r)\right)}+\frac{t a(r) h_{j}^{\prime}(r)}{i\left(x \cdot \omega+t h_{j}(r)\right)^{2}} .
$$

If $|\omega|=1$, we obtain that

$$
\frac{1}{2}\left|t h_{j}(r)\right| \leq\left|t h_{j}(r)\right|-|x| \leq\left|t h_{j}(r)+x \cdot \omega\right| .
$$

Then, using previous inequality and condition [H] for $n=$ 2 , we concluded that there is $C>0$ (independent of $j$ ) such that

$$
\begin{aligned}
\left|\left(K_{j}(t)\right)(x)\right| & \leq C \int_{S^{1}}|I| d S_{\omega} \\
& \leq C \int_{S^{1}} \frac{1}{\left|h_{j}(r)\right|}\left(1+\frac{\left|h_{j}^{\prime}(r)\right|}{\left|h_{j}(r)\right|}\right) \frac{1}{|t|} d S_{\omega} \\
& \leq \frac{C}{|t|}
\end{aligned}
$$

Applying the same argument of integration by parts as before, it follows that

$$
I=\int_{0}^{\infty} e^{i r\left(x \cdot \omega+t \rho_{j}(r)\right)} G(r) d r
$$

where

$$
\begin{aligned}
G(r) & =\frac{-a^{\prime \prime}(r)}{\left(x \cdot \omega+t h_{j}(r)\right)^{2}}-\frac{3 t a^{\prime}(r) h_{j}^{\prime}(r)}{\left(x \cdot \omega+t h_{j}(r)\right)^{3}} \\
& +\frac{t a(r) h_{j}^{\prime \prime}(r)}{\left(x \cdot \omega+t h_{j}(r)\right)^{3}}-\frac{3 t^{2} a(r)\left(h_{j}^{\prime}(r)\right)^{2}}{\left(x \cdot \omega+t h_{j}(r)\right)^{4}} .
\end{aligned}
$$

Then, using inequality (8) and condition $[\mathbf{H}]$ for $n=3$, we obtain that

$|I| \leq$

$\frac{C}{\left|h_{j}(r)\right|^{2}}\left(1+\frac{\left|h_{j}(r) h_{j}^{\prime}(r)\right|+\left|h_{j}(r) h_{j}^{\prime \prime}(r)\right|+\left|h_{j}^{\prime}(r)\right|^{2}}{\left|h_{j}(r)\right|^{2}}\right) \frac{1}{|t|^{2}}$

$\leq \frac{C}{|t|^{2}}$.

If we continue using the integration by part argument and condition $[\mathbf{H}]$, we have after the $n-1$ step that

$$
|I| \leq \frac{C_{n}}{\left|h_{j}(r)\right|^{n-1}}\left(1+\frac{\sum_{k} f_{k}}{\left|h_{j}(r)\right|^{n-1}}\right) \frac{1}{|t|^{n-1}} \leq \frac{C_{n}}{|t|^{n-1}} .
$$

where the sum is finite and each term $f_{k}$ is the product of $n-1$ functions in the set

$$
\left\{h_{j}, h_{j}^{\prime}, \ldots, h_{j}^{(n-1)}\right\} \text {. }
$$

In other words,

$$
\left|\left(K_{j}(t)\right)(x)\right| \leq C \int_{S^{n-1}}|I| d S_{\omega} \leq \frac{C_{n}}{|t|^{n-1}} .
$$

Then we see that (7) follows by combining case 1 . and case 2.

Lemma 6. Let $n \geq 2$ and assume that $\rho$ satisfies $[\mathbf{H}]$. If $2 \leq p \leq \infty$ and $\frac{1}{p}+\frac{1}{p^{\prime}}=1$, then there exist $C>0$ such that for all $j \in \mathbb{Z}$,

$$
\left\|T_{j}(t) f\right\|_{p} \leq C|t|^{\frac{-(n-1)}{2}\left(\frac{1}{p^{\prime}}-\frac{1}{p}\right)}\|f\|_{p^{\prime}} .
$$

Proof. Using the Riesz-Thorin Interpolation Theorem and previous lemma we obtain that

$$
T_{j}(t): L^{p^{\prime}}\left(\mathbb{R}^{n}\right) \rightarrow L^{p}\left(\mathbb{R}^{n}\right)
$$

is a bounded linear operator and there is a positive constant $C$ (independent of $j$ ) such that

$$
\left\|T_{j}(t) f\right\|_{p} \leq C\left(|t|^{\frac{-(n-1)}{2}}\right)^{1-\theta}\|f\|_{p^{\prime}}
$$

where

$$
\frac{1}{p}=\frac{\theta}{2} \quad \text { and } \quad 1-\theta=1-\frac{2}{p}=\frac{1}{p^{\prime}}-\frac{1}{p},
$$

as desired.

Hereafter we will assume, unless otherwise stated, that $2 \leq p<\infty, 2 \leq q \leq \infty$ and $n \geq 2$ satisfy the relation

$$
\frac{2}{q}=\frac{n-1}{2}\left(1-\frac{2}{p}\right) \text {. }
$$

We will say that the couple $(p, q)$ is an admissible pair if satisfies (9) for a fixed $n \geq 2$. The following notation also is used,

$$
\frac{1}{p}+\frac{1}{p^{\prime}}=\frac{1}{q}+\frac{1}{q^{\prime}}=1
$$


Lemma 7. Assume that $\rho$ satisfies [H]. If $2<p<\infty$ and $(p, q)$ is an admissible pair, then there exist $C>0$ such that for all $j \in \mathbb{Z}$,

$$
\begin{aligned}
& \left(\int_{-\infty}^{\infty}\left\|\int_{-\infty}^{\infty} T_{j}(t-r) g(\cdot, r) d r\right\|_{p}^{q} d t\right)^{1 / q} \\
& \leq C\left(\int_{-\infty}^{\infty}\|g(\cdot, t)\|_{p^{\prime}}^{q^{\prime}} d t\right)^{1 / q^{\prime}}, \\
& \left\|\int_{-\infty}^{\infty} T_{j}(t) g(\cdot, t) d t\right\|_{2} \leq C\left(\int_{-\infty}^{\infty}\|g(\cdot, t)\|_{p^{\prime}}^{q^{\prime}} d t\right)^{1 / q^{\prime}}, \\
& \left(\int_{-\infty}^{\infty}\left\|T_{j}(t) f\right\|_{p}^{q} d t\right)^{1 / q} \leq C\|f\|_{2},
\end{aligned}
$$

where $C=C_{p, n}$ is a constant that depends only on $p$ and $n$.

Proof. First we will show (10). Using the Minkowski integral inequality and previous proposition we have that

$$
\begin{aligned}
& \left\|\int_{-\infty}^{\infty} T_{j}(t-r) g(\cdot, r) d r\right\|_{p} \\
& \leq \int_{-\infty}^{\infty}\left\|T_{j}(t-r) g(\cdot, r)\right\|_{p} d r \\
& \leq C \int_{-\infty}^{\infty}|t-r|^{\frac{-(n-1)}{2}\left(\frac{1}{p^{\prime}}-\frac{1}{p}\right)}\|g(\cdot, r)\|_{p^{\prime}} d r .
\end{aligned}
$$

From the Hardy-Littlewood-Sobolev inequality we obtain (10) with

$$
\frac{1}{q}=\frac{1}{q^{\prime}}-\left[1-\frac{n-1}{2}\left(\frac{1}{p^{\prime}}-\frac{1}{p}\right)\right] .
$$

This implies that

$$
\frac{2}{q}=\frac{(n-1)}{2}\left(1-\frac{2}{p}\right) .
$$

Now, define $h(x)=\int_{-\infty}^{\infty}\left(T_{j}(t) g(\cdot, t)\right)(x) d t$. Then we obtain that

$$
\begin{aligned}
& \|h\|_{L^{2}\left(\mathbb{R}^{n}\right)}^{2} \\
& =\int_{\mathbb{R}^{n}}\left[h(x)\left(\int_{-\infty}^{\infty}\left(T_{j}(-r) \overline{g(\cdot, r)}\right)(x) d r\right)\right] d x \\
& =\int_{-\infty}^{\infty} \int_{\mathbb{R}^{n}}\left[g(x, t) \int_{-\infty}^{\infty}\left(T_{j}(t-r) \overline{g(\cdot, r)}\right)(x) d r\right] d x d t \\
& \leq\left(\int_{-\infty}^{\infty}\|g(\cdot, t)\|_{p^{\prime}}^{q^{\prime}} d t\right)^{1 / q^{\prime}} \times \\
& \times\left(\int_{-\infty}^{\infty}\left\|\int_{-\infty}^{\infty} T_{j}(t-r) \overline{g(\cdot, r)} d r\right\|_{p}^{q} d t\right)^{1 / q} .
\end{aligned}
$$

Hence, using (10) we find that

$$
\|h\|_{L^{2}\left(\mathbb{R}^{n}\right)}^{2} \leq C\left(\int_{-\infty}^{\infty}\|g(\cdot, t)\|_{p^{\prime}}^{q^{\prime}} d t\right)^{2 / q^{\prime}}
$$

which implies (11). To finish the proof we see that a duality argument implies that

$$
\begin{aligned}
& \left(\int_{-\infty}^{\infty}\|h(x, t)\|_{p}^{q} d t\right)^{1 / q} \\
& =\sup \left\{\left|\int_{-\infty}^{\infty} \int_{\mathbb{R}^{n}} h(x, t) w(x, t) d x d t\right|:\|w\|_{L_{t}^{q^{\prime}}\left(L_{x}^{p^{\prime}}\right)}=1\right\} .
\end{aligned}
$$

Then if $\|w\|_{L_{t}^{q^{\prime}}\left(L_{x}^{p^{\prime}}\right)}=1$,

$$
\begin{aligned}
& \left|\int_{-\infty}^{\infty} \int_{\mathbb{R}^{n}}\left(T_{j}(t) f\right)(x) w(x, t) d x d t\right| \\
& \leq\|f\|_{2}\left\|\int_{-\infty}^{\infty}\left(T_{j}(t) w(\cdot, t)\right)(x) d t\right\|_{2} \\
& \leq\|f\|_{2}\|w\|_{L_{t}^{q^{\prime}}\left(L_{x}^{p^{\prime}}\right)} \\
& \leq\|f\|_{2} .
\end{aligned}
$$

So that (12) follows, as desired.

\subsection{Global estimates}

Now, using the local estimates, we are position to obtain Strichartz estimates for the semigroups $S_{k}(k=1,2,3)$, given by the equation (3) with $\varphi_{k} \in L^{\infty}\left(\mathbb{R}^{n}\right)$ and the function $\rho: \mathbb{R} \rightarrow \mathbb{R}$ satisfying the condition $[\mathbf{H}]$. Our argument is based on the Littlewood-Paley dyadic ecomposition, the estimate (12) and Lemma 6.

Theorem 1. Assume that $\varphi_{k} \in L^{\infty}\left(\mathbb{R}^{n}\right)$ for $1 \leq k \leq 3$ and that $\rho$ satisfies the condition $[\mathbf{H}]$. If $(p, q)$ is an admissible pair and $\alpha=\frac{n}{2}-\frac{n}{p}-\frac{1}{q}$, then there exist $C_{k}>0$ such that

$$
\begin{aligned}
& \text { 1) }\left\|S_{1}(t) f\right\|_{L_{t}^{q}\left(L_{x}^{p}\right)} \leq C_{1}\|f\|_{\dot{H}^{\alpha}\left(\mathbb{R}^{n}\right)}, \\
& \text { 2) }\left\|S_{2}(t) f\right\|_{L_{t}^{q}\left(L_{x}^{p}\right)} \leq C_{2}\|f\|_{\dot{H}^{\alpha+1}\left(\mathbb{R}^{n}\right)}, \\
& \text { 3) }\left\|S_{3}(t) f\right\|_{L_{t}^{q}\left(L_{x}^{p}\right)} \leq C_{3}\|f\|_{\dot{H}^{\alpha-1}\left(\mathbb{R}^{n}\right)} .
\end{aligned}
$$

Proof. First we consider the operator $U(t)$ defined in Fourier space by

$$
\widehat{U(t) f}(\xi)=e^{i t|\xi| \rho(|\xi|)} \widehat{f}(\xi),
$$

and use the Littlewood-Paley decomposition $\left\{\Delta_{j} f\right\}$ given by

$$
\widehat{\Delta_{j} f}(\xi)=\beta\left(\frac{\xi}{2^{j}}\right) \widehat{f}(\xi), \quad j \in \mathbb{Z},
$$

where $\beta \in C_{0}^{\infty}\left(\mathbb{R}^{n}\right)$ is a radial function supported away from zero. Then we have that $f=\sum_{j \in \mathbb{Z}} \Delta_{j} f$. Moreover,

$$
U(t) f=\sum_{j \in \mathbb{Z}} \Delta_{j}(U(t) f)=\sum_{j \in \mathbb{Z}} U(t)\left(\Delta_{j} f\right) .
$$


Now, we see that

$$
\begin{aligned}
& \left(U(t) \Delta_{j} f\right)(x) \\
& =\frac{1}{(2 \pi)^{\frac{n}{2}}} \int_{\mathbb{R}^{n}} e^{i x \cdot \xi} e^{i t|\xi| \rho(|\xi|)} \beta\left(\frac{\xi}{2^{j}}\right) \widehat{f}(\xi) d \xi \\
& =\frac{1}{(2 \pi)^{\frac{n}{2}}} \int_{\mathbb{R}^{n}} e^{i\left(2^{j} x\right) \cdot \xi} e^{i\left(2^{j} t\right)|\xi| \rho\left(2^{j}|\xi|\right)} \beta(\xi) \widehat{g}(\xi) d \xi,
\end{aligned}
$$

where $g(\cdot)=f\left(\frac{\dot{2}}{2^{j}}\right)$. Then we conclude that

$$
\left(U(t) \Delta_{j} f\right)(x)=\left(T_{j}(g)\right)\left(2^{j} x, 2^{j} t\right), \quad(x, t) \in \mathbb{R}^{n+1} .
$$

Using this fact and inequality (12) for $2<p<\infty$ and

$$
\frac{2}{q}=\frac{n-1}{2}\left(1-\frac{2}{p}\right)
$$

we find that

$$
\begin{aligned}
\left\|U(t) \Delta_{j} f\right\|_{L_{t}^{q}\left(L_{x}^{p}\right)} & =2^{j\left(-\frac{n}{p}-\frac{1}{q}\right)}\left\|T_{j}(g)\right\|_{L_{t}^{q}\left(L_{x}^{p}\right)} \\
& \leq C 2^{j\left(-\frac{n}{p}-\frac{1}{q}\right)}\|g\|_{2} \\
& \leq C 2^{j\left(\frac{n}{2}-\frac{n}{p}-\frac{1}{q}\right)}\|f\|_{2} .
\end{aligned}
$$

By orthogonality property (2), for fixed $j$ we have that

$$
\Delta_{j} f=\Delta_{j}\left(\sum_{l \in \mathbb{Z}} \Delta_{l} f\right)=\sum_{l \in \mathbb{Z},|l-j| \leq 3} \Delta_{j} \Delta_{l} f .
$$

Then if $\alpha=\frac{n}{2}-\frac{n}{p}-\frac{1}{q}$ we see from (14) and Bernstein's Lemma that

$$
\begin{aligned}
\left\|U(t) \Delta_{j} f\right\|_{L_{t}^{q}\left(L_{x}^{p}\right)} & \leq \sum_{|l-j| \leq 3}\left\|U(t) \Delta_{j} \Delta_{l} f\right\|_{L_{t}^{q}\left(L_{x}^{p}\right)} \\
& \leq C \sum_{|l-j| \leq 3} 2^{j \alpha}\left\|\Delta_{l} f\right\|_{2} \\
& \leq C_{\alpha} \sum_{|l-j| \leq 3}\left\|\Delta_{l} f\right\|_{\dot{H}^{\alpha}\left(\mathbb{R}^{n}\right)} .
\end{aligned}
$$

Hence, using Lemma 2 and previous inequality we find that

$$
\begin{aligned}
\|U(t) f\|_{L_{t}^{q}\left(L_{x}^{p}\right)} & \leq\left\|\sqrt{\sum_{j \in \mathbb{Z}}\left\|U(t) \Delta_{j} f\right\|_{L_{x}^{p}}^{2}}\right\|_{L_{t}^{q}} \\
& \leq \sqrt{\sum_{j \in \mathbb{Z}}\left\|U(t) \Delta_{j} f\right\|_{L_{t}^{q}\left(L_{x}^{p}\right)}^{2}} \\
& \leq C_{\alpha} \sqrt{\sum_{j \in \mathbb{Z}|l-j| \leq 3} \sum_{l}\left\|\Delta_{l} f\right\|_{\dot{H}^{\alpha}\left(\mathbb{R}^{n}\right)}^{2}} \\
& \leq C_{\alpha} \sqrt{\sum_{j \in \mathbb{Z}}\left\|\Delta_{j} f\right\|_{\dot{H}^{\alpha}\left(\mathbb{R}^{n}\right)}^{2}} \\
& \leq C_{\alpha}\|f\|_{\dot{H}^{\alpha}\left(\mathbb{R}^{n}\right)} .
\end{aligned}
$$

Now, if $S_{1}(t)$ is given by

$$
\widehat{S_{1}(t) f}(\xi)=\varphi_{1}(\xi) e^{i t|\xi| \rho(|\xi|)} \widehat{f}(\xi),
$$

we set $\widehat{g}_{1}(\xi)=\varphi_{1}(\xi) \widehat{f}(\xi)$. Then using (15) we have that

$$
\begin{aligned}
\left\|S_{1}(t) f\right\|_{L_{t}^{q}\left(L_{x}^{p}\right)} & =\left\|U(t) g_{1}\right\|_{L_{t}^{q}\left(L_{x}^{p}\right)} \\
& \leq C_{\alpha}\left\|g_{1}\right\|_{\dot{H}^{\alpha}\left(\mathbb{R}^{n}\right)} \\
& \leq C_{\alpha}\|\varphi\|_{\infty}\|f\|_{\dot{H}^{\alpha}\left(\mathbb{R}^{n}\right)} .
\end{aligned}
$$

Recall that (14) holds for $2<p<\infty$. We notice that if $p=2$, then $q=\infty, \alpha=0$ and $\dot{H}^{\alpha}\left(\mathbb{R}^{n}\right)=L^{2}\left(\mathbb{R}^{n}\right)$. In this case for all $t \in \mathbb{R}$ we see that

$$
\left\|S_{1}(t) f\right\|_{2}=\left\|\widehat{S_{1}(t) f}\right\|_{2} \leq\left\|\varphi_{1}\right\|_{\infty}\|f\|_{2} .
$$

Therefore

$$
\left\|S_{1}(t) f\right\|_{L_{t}^{\infty}\left(L_{x}^{2}\right)} \leq C\|f\|_{2} .
$$

Using $\widehat{g}_{2}(\xi)=|\xi| \varphi_{2}(\xi) \widehat{f}(\xi)$ and $\widehat{g}_{3}(\xi)=\varphi_{3}(\xi) \widehat{f}(\xi) /|\xi|$ we obtain the statements 2 ) and 3 ) of theorem.

Corollary 1. Assume that $\varphi_{k} \in L^{\infty}\left(\mathbb{R}^{n}\right)$ for $1 \leq k \leq 3$ and that $\rho$ satisfies the condition $[\mathbf{H}]$. If $s \in \mathbb{R},(p, q)$ is an admissible pair and $\alpha=\frac{n}{2}-\frac{n}{p}-\frac{1}{q}$, then there exist $C_{k}>0$ such that

$$
\begin{aligned}
& \text { 1) }\left\|S_{1}(t) f\right\|_{L_{t}^{q}\left(\dot{H}^{s, p}\left(\mathbb{R}^{n}\right)\right)} \leq C_{1}\|f\|_{\dot{H}^{s+\alpha}\left(\mathbb{R}^{n}\right)}, \\
& \text { 2) }\left\|S_{2}(t) f\right\|_{L_{t}^{q}\left(\dot{H}^{s, p}\left(\mathbb{R}^{n}\right)\right)} \leq C_{2}\|f\|_{\dot{H}^{s+\alpha+1}\left(\mathbb{R}^{n}\right)} \text {, } \\
& \text { 3) }\left\|S_{3}(t) f\right\|_{L_{t}^{q}\left(\dot{H}^{s, p}\left(\mathbb{R}^{n}\right)\right)} \leq C_{3}\|f\|_{\dot{H}^{s+\alpha-1}\left(\mathbb{R}^{n}\right)} .
\end{aligned}
$$

Proof. Let $s \in \mathbb{R}$ and define $\widehat{g}(\xi)=|\xi|^{s} \widehat{f}(\xi)$, then using Theorem 1 we have that

$$
\begin{aligned}
\left(\int_{-\infty}^{\infty}\left\|S_{1}(t) f\right\|_{\dot{H}^{s, p}\left(\mathbb{R}^{n}\right)}^{q} d t\right)^{1 / q} & =\left(\int_{-\infty}^{\infty}\left\|S_{1}(t) g\right\|_{p}^{q} d t\right)^{1 / q} \\
& \leq C\|g\|_{\dot{H}^{\alpha}\left(\mathbb{R}^{n}\right)} \\
& =C\|f\|_{\dot{H}^{s+\alpha}\left(\mathbb{R}^{n}\right)} .
\end{aligned}
$$

We use the obvious modification when $q=\infty$. Similarly follows 2) and 3).

Theorem 2. Assume that $\varphi_{k} \in L^{\infty}\left(\mathbb{R}^{n}\right)$ for $1 \leq k \leq 3$ and that $\rho$ satisfies the condition $[\mathbf{H}]$. If $s \in \mathbb{R}, 2<p<\infty$ and $\gamma=\frac{n+1}{2}\left(\frac{1}{p^{\prime}}-\frac{1}{p}\right)$, then there exist $C_{k}>0$ such that

$$
\begin{aligned}
& \text { 1) }\left\|S_{1}(t) f\right\|_{\dot{H}^{s, p}\left(\mathbb{R}^{n}\right)} \leq C_{1}|t|^{\frac{-(n-1)}{2}\left(\frac{1}{p^{\prime}}-\frac{1}{p}\right)}\|f\|_{\dot{H}^{s+\gamma, p^{\prime}}\left(\mathbb{R}^{n}\right)^{\prime}} . \\
& \text { 2) }\left\|S_{2}(t) f\right\|_{\dot{H}^{s, p}\left(\mathbb{R}^{n}\right)} \leq C_{2}|t|^{\frac{-(n-1)}{2}\left(\frac{1}{p^{\prime}}-\frac{1}{p}\right)}\|f\|_{\dot{H}^{s+\gamma+1, p^{\prime}}\left(\mathbb{R}^{n}\right)} \text {. } \\
& \text { 3) }\left\|S_{3}(t) f\right\|_{\dot{H}^{s, p}\left(\mathbb{R}^{n}\right)} \leq C_{3}|t|^{\frac{-(n-1)}{2}\left(\frac{1}{p^{\prime}}-\frac{1}{p}\right)}\|f\|_{\dot{H}^{s+\gamma-1, p^{\prime}}\left(\mathbb{R}^{n}\right)^{\prime}} .
\end{aligned}
$$

Proof. For the proof of 1 ) it is suffices to show that

$$
\left\|S_{1}(t) f\right\|_{p} \leq C|t|^{\frac{-(n-1)}{2}\left(\frac{1}{p^{\prime}}-\frac{1}{p}\right)}\|f\|_{\dot{H}^{\gamma, p^{\prime}}\left(\mathbb{R}^{n}\right)} .
$$

Using the same notation as in the proof of Theorem 1 we have that

$$
\left(U(t) \Delta_{j} f\right)(x)=\left(T_{j}(g)\right)\left(2^{j} x, 2^{j} t\right), \quad(x, t) \in \mathbb{R}^{n+1},
$$


where $g(\cdot)=f\left(\frac{\dot{2}}{2^{j}}\right)$. Then using Lemma 6 for $2<p<\infty$ we find that

$$
\left\|U(t) \Delta_{j} f\right\|_{p} \leq C 2^{j \gamma}|t|^{\frac{-(n-1)}{2}}\left(\frac{1}{p^{\prime}}-\frac{1}{p}\right)\|f\|_{p^{\prime}}
$$

From this inequality and proceeding as in the proof of Theorem 1 the result follows.

\section{Strichartz estimates for some water wave models}

\subsection{The generalized Benney-Luke equation}

In this section we establish Strichartz estimates for the semigroup associated with the two-dimensional generalized Benney-Luke (gBL) equation

$$
\begin{aligned}
\Phi_{t t}-\Delta \Phi & +\mu\left(a \Delta^{2} \Phi-b \Delta \Phi_{t t}\right) \\
& +\varepsilon\left[\Phi_{t}\left(\left[\left(\Phi_{x}\right)^{m}\right]_{x}+\left[\left(\Phi_{y}\right)^{m}\right]_{y}\right)\right. \\
& \left.+2\left(\left(\Phi_{x}\right)^{m} \Phi_{x t}+\left(\Phi_{y}\right)^{m} \Phi_{y t}\right)\right]=0,
\end{aligned}
$$

where $a, b, \varepsilon, \mu>0$ and $m=\frac{m_{1}}{m_{2}}$ with $\left(m_{1}, m_{2}\right)=1$ and $m_{2}$ odd. J. Quintero and R. Pego in [14] showed that the evolution of three-dimensional water waves with surface tension can be reduced to studying the solution $\Phi(x, y, t)$ of the equation (16) with $m=1$, where $\varepsilon$ is the amplitude parameter, $\mu$ is the long-wave parameter and $a-b=\sigma-\frac{1}{3}$, where $\sigma$ is the Bond number (associated with the surface tension). The variable $\Phi$ is the rescale nondimensional velocity potential on the bottom $z=0$.

Hereafter we consider $\varepsilon=\mu=1$, since the parameters $\varepsilon$ and $\mu$ can be scale from the equation by defining

$$
\widetilde{\Phi}(x, y, t)=\frac{\sqrt{\mu}}{\varepsilon} \Phi\left(\frac{x}{\sqrt{\mu}}, \frac{y}{\sqrt{\mu}}, \frac{y}{\sqrt{\mu}}\right) .
$$

Also, we will writte $H^{s, p}=H^{s, p}\left(\mathbb{R}^{2}\right), \dot{H}^{s, p}=\dot{H}^{s, p}\left(\mathbb{R}^{2}\right)$ and $\mathcal{V}^{s}=\mathcal{V}^{s}\left(\mathbb{R}^{2}\right)$.

We observe that the generalized Benney-Luke equation can be written as a first-order system in the variables $\Phi$ and $w=\Phi_{t}$,

$$
\left(\begin{array}{l}
\Phi \\
w
\end{array}\right)_{t}+M\left(\begin{array}{l}
\Phi \\
w
\end{array}\right)+G\left(\begin{array}{l}
\Phi \\
w
\end{array}\right)=0
$$

where

$$
M=\left(\begin{array}{cc}
0 & I \\
\Delta B^{-1} A & 0
\end{array}\right)
$$

with $A=I-a \Delta, B=I-b \Delta$, where $G$ and $F$ are given by

$$
\begin{aligned}
G\left(\begin{array}{c}
\Phi \\
w
\end{array}\right) & =\left(\begin{array}{c}
0 \\
B^{-1} F\left(\Phi_{x}, \Phi_{y}, w\right)
\end{array}\right), \\
F(u, v, w) & =\left[w\left(\left(u^{m}\right)_{x}+\left(v^{m}\right)_{y}\right)+2\left(u^{m} w_{x}+v^{m} w_{y}\right)\right] .
\end{aligned}
$$

We see that the Fourier symbol associated with the operators $A$ and $B$ are given by

$$
\widehat{A}=1+a|\xi|^{2}, \widehat{B}=1+b|\xi|^{2}
$$

We see directly that the linear operator $M$ is the infinitesimal generator of a $C_{0}$-semigroup $S(t)$. In fact, $S(t)$ is defined as

$$
S(t)=\left(\begin{array}{cc}
\mathcal{F}^{-1} & 0 \\
0 & \mathcal{F}^{-1}
\end{array}\right) \widehat{e^{t M}}\left(\begin{array}{cc}
\mathcal{F} & 0 \\
0 & \mathcal{F}
\end{array}\right)
$$

where we have that

$$
\widehat{e^{t M}}=\left(\begin{array}{cc}
\cos (|\xi| \rho(|\xi|) \mid t) & \frac{\sin (|\xi| \rho(|\xi|) t)}{|\xi| \rho(|\xi|)} \\
-|\xi| \rho(|\xi|) \sin (|\xi| \rho(|\xi|) t) & \cos (|\xi| \rho(|\xi|) t)
\end{array}\right)
$$

with $\rho$ being defined as

$$
\rho(r)=\sqrt{\frac{1+a r^{2}}{1+b r^{2}}} .
$$

Lemma 8. $\rho$ satisfies the condition $[\mathbf{H}]$.

Proof. Since $n=2$, we want to establish that

$$
\left|h_{j}(r)\right| \geq c_{0}, \quad\left|h_{j}^{\prime}(r)\right| \leq c_{1},
$$

which implies the condition [H]. If $\rho_{j}(r)=\rho\left(2^{j} r\right)$, we have that

$$
\begin{aligned}
h_{j}(r) & =\rho_{j}(r)+r \rho_{j}^{\prime}(r) \\
& =\sqrt{\frac{1+4^{j} a r^{2}}{1+4^{j} b r^{2}}}+\frac{(a-b) 4^{j} r^{2}}{\left(1+4^{j} b r^{2}\right)^{2}} \sqrt{\frac{1+4^{j} b r^{2}}{1+4^{j} a r^{2}}} \\
& =\frac{1+(2 a+b) 4^{j} r^{2}+3 a b 4^{2 j} r^{4}+a b^{2} 4^{3 j} r^{6}}{\left(1+4^{j} b r^{2}\right)^{2} \sqrt{\left(1+4^{j} a r^{2}\right)\left(1+4^{j} b r^{2}\right)}}
\end{aligned}
$$

Let $I=\left[r_{1}, r_{2}\right] \subset \mathbb{R}^{+}$, then for any $(j, r) \in \mathbb{Z} \times I$ we have the estimate

$$
h_{j}(r) \geq \frac{1+(2 a+b) 4^{j} r_{1}^{2}+3 a b 4^{2 j} r_{1}^{4}+a b^{2} 4^{3 j} r_{1}^{6}}{\left(1+4^{j} b r_{2}^{2}\right)^{2} \sqrt{\left(1+4^{j} a r_{2}^{2}\right)\left(1+4^{j} b r_{2}^{2}\right)}}:=\pi_{j}
$$


Now, we have that

$$
\lim _{j \rightarrow-\infty} \pi_{j}=1, \quad \lim _{j \rightarrow \infty} \pi_{j}=\sqrt{\frac{a}{b}}\left(\frac{r_{1}}{r_{2}}\right)^{6} .
$$

Hence, we concluded that there exist $c_{0}>0$ such that for all $(j, r) \in \mathbb{Z} \times I$,

$$
\left|h_{j}(r)\right| \geq c_{0} .
$$

On the other hand, we have that

$$
\begin{aligned}
h_{j}^{\prime}(r)= & \frac{2(2 a+b) 4^{j} r+12 a b 4^{2 j} r^{3}+6 a b^{2} 4^{3 j} r^{5}}{\left(1+b 4^{j} r^{2}\right)^{2} \sqrt{\left(1+a 4^{j} r^{2}\right)\left(1+b 4^{j} r^{2}\right)}} \\
- & \frac{4 b\left(1+(2 a+b) 4^{j} r^{2}+3 a b 4^{2 j} r^{4}+a b^{2} 4^{3 j} r^{6}\right) 4^{j} r}{\left(1+b 4^{j} r^{2}\right)^{3} \sqrt{\left(1+a 4^{j} r^{2}\right)\left(1+b 4^{j} r^{2}\right)}} \\
- & \frac{\left((a+b) 4^{j} r+2 a b 4^{2 j} r^{3}\right)}{\left(1+a 4^{j} r^{2}\right)\left(1+b 4^{j} r^{2}\right)^{3}} \times \\
& \times \frac{\left(1+(2 a+b) 4^{j} r^{2}+3 a b 4^{2 j} r^{4}+a b^{2} 4^{3 j} r^{6}\right)}{\sqrt{\left(1+a 4^{j} r^{2}\right)\left(1+b 4^{j} r^{2}\right)}} .
\end{aligned}
$$

Then for any $(j, r) \in \mathbb{Z} \times I$ we find that

$$
\begin{aligned}
\left|h_{j}^{\prime}(r)\right| \leq & \frac{2(2 a+b) 4^{j} r_{1}+12 a b 4^{2 j} r_{1}^{3}+6 a b^{2} 4^{3 j} r_{1}^{5}}{\left(1+b 4^{j} r_{2}^{2}\right)^{2} \sqrt{\left(1+a 4^{j} r_{2}^{2}\right)\left(1+b 4^{j} r_{2}^{2}\right)}} \\
+ & \frac{4 b\left(1+(2 a+b) 4^{j} r_{1}^{2}+3 a b 4^{2 j} r_{1}^{4}+a b^{2} 4^{3 j} r_{1}^{6}\right) 4^{j} r}{\left(1+b 4^{j} r_{2}^{2}\right)^{3} \sqrt{\left(1+a 4^{j} r_{2}^{2}\right)\left(1+b 4^{j} r_{2}^{2}\right)}} \\
+ & \frac{\left((a+b) 4^{j} r+2 a b 4^{2 j} r^{3}\right)}{\left(1+a 4^{j} r^{2}\right)\left(1+b 4^{j} r^{2}\right)^{3}} \times \\
& \times \frac{\left(1+(2 a+b) 4^{j} r^{2}+3 a b 4^{2 j} r^{4}+a b^{2} 4^{3 j} r^{6}\right)}{\sqrt{\left(1+a 4^{j} r^{2}\right)\left(1+b 4^{j} r^{2}\right)}} .
\end{aligned}
$$

Then we see that

$$
\lim _{j \rightarrow-\infty} h_{j}^{\prime}(r)=\lim _{j \rightarrow \infty} h_{j}^{\prime}(r)=0 .
$$

So that, there exist $c_{1}>0$ such that $\left|h_{j}^{\prime}(r)\right| \leq c_{1}$ for all $(j, r) \in \mathbb{Z} \times I$. Hence, for $c_{2}=\frac{c_{1}}{c_{0}}$ we conclude that

$$
\frac{\left|h_{j}^{\prime}(r)\right|}{\left|h_{j}(r)\right|} \leq c_{2} \text {. }
$$

Next, we will state the Strichartz estimates for the semigroup $S(t)$ associated with the system (17).
Theorem 3. Suppose $s \in \mathbb{R}, 2 \leq p<\infty, \frac{1}{q}=\frac{1}{4}-\frac{1}{2 p}$ and $\alpha=\frac{3}{4}-\frac{3}{2 p}$. Then the semigroup $S(t)$ satisfies the estimates $\|S(t)(\Phi, w)\|_{L_{t}^{q}\left(\dot{H}^{s, p} \times \dot{H}^{s-1, p}\right)} \leq C\left(\|\Phi\|_{\dot{H}^{s+\alpha}}+\|w\|_{\dot{H}^{s+\alpha-1}}\right)$.

Proof. From the previous lemma we have that $\pm \rho(r)$ satisfies the condition $[\mathbf{H}]$. Moreover, the functions $\rho(|\xi|), 1 / \rho(|\xi|)$ are bounded and the functions $\sin (|\xi| \rho(|\xi|) t)$ and $\cos (|\xi| \rho(|\xi|) t)$ are linear combinations of $e^{ \pm i|\xi| \rho(|\xi|) t}$. Then using Corollary 1 for $n=2$, we have that

$$
\begin{aligned}
& \left\|\mathcal{F}^{-1}(\cos (|\xi| \rho(|\xi|) t) \widehat{\Phi})\right\|_{L_{t}^{q}\left(\dot{H}^{s, p}\right)} \\
& \quad+\left\|\mathcal{F}^{-1}\left(\frac{\sin (|\xi| \rho(|\xi|) t)}{|\xi| \rho(|\xi|)} \widehat{w}\right)\right\|_{L_{t}^{q}\left(\dot{H}^{s, p}\right)} \\
& \quad \leq C\left(\|\Phi\|_{\dot{H}^{s+\alpha}}+\|w\|_{\dot{H}^{s+\alpha-1}}\right),
\end{aligned}
$$

and also that

$$
\begin{aligned}
& \left\|\mathcal{F}^{-1}(|\xi| \rho(|\xi|) \sin (|\xi| \rho(|\xi|) t) \widehat{\Phi})\right\|_{L_{t}^{q}\left(\dot{H}^{s-1, p}\right)} \\
& +\left\|\mathcal{F}^{-1}(\cos (|\xi| \rho(|\xi|) t) \widehat{w})\right\|_{L_{t}^{q}\left(\dot{H}^{s-1, p}\right)} \\
& \leq C\left(\|\Phi\|_{\dot{H}^{s+\alpha}}+\|w\|_{\dot{H}^{s+\alpha-1}}\right) .
\end{aligned}
$$

Then we obtain that

$\|S(t)(\Phi, w)\|_{L_{t}^{q}\left(\dot{H}^{s, p} \times \dot{H}^{s-1, p}\right)} \leq C\left(\|\Phi\|_{\dot{H}^{s+\alpha}}+\|w\|_{\dot{H}^{s+\alpha-1}}\right)$.

In a similar fashion, using Theorem 2 , also we have the following estimates for the semigroup $S(t)$.

Theorem 4. Suppose $s \in \mathbb{R}, 2<p<\infty, \frac{1}{p}+\frac{1}{p^{\prime}}=1$ and $\gamma=\frac{3}{2}\left(\frac{1}{p^{\prime}}-\frac{1}{p}\right)$. Then there exist $C>0$ such that

$$
\begin{aligned}
& \|S(t)(\Phi, w)\|_{\dot{H}^{s, p} \times \dot{H}^{s-1, p}} \\
& \quad \leq C|t|^{\frac{-1}{2}\left(\frac{1}{p^{\prime}}-\frac{1}{p}\right)}\left(\|\Phi\|_{\dot{H}^{s+\gamma, p^{\prime}}}+\|w\|_{\dot{H}^{s+\gamma-1, p^{\prime}}}\right) .
\end{aligned}
$$

Now we have the following classical results for the linear wave equation in $\mathbb{R}^{n}$.

Corollary 2. Suppose $n \geq 2, s \in \mathbb{R},(p, q)$ an admissible pair and $\alpha=\frac{n}{2}-\frac{2}{p}-\frac{1}{q}$. If $\Phi_{0} \in \dot{H}^{s+\alpha}\left(\mathbb{R}^{n}\right)$, $w_{0} \in \dot{H}^{s+\alpha-1}\left(\mathbb{R}^{n}\right)$ and $\Phi$ is a solution to the initial value problem in $\mathbb{R}^{n}$,

$$
\begin{gathered}
\Phi_{t t}-\Delta \Phi=0 \\
\Phi(x, 0)=\Phi_{0}(x), \quad \Phi_{t}(x, 0)=w_{0}(x) .
\end{gathered}
$$

Then the following estimate holds

$$
\begin{aligned}
\|\Phi\|_{L_{t}^{q}\left(\dot{H}^{s, p}\left(\mathbb{R}^{n}\right)\right)} & +\left\|\Phi_{t}\right\|_{L_{t}^{q}\left(\dot{H}^{s-1, p}\left(\mathbb{R}^{n}\right)\right)} \\
\leq & C\left(\left\|\Phi_{0}\right\|_{H^{s+\alpha}\left(\mathbb{R}^{n}\right)}+\left\|w_{0}\right\|_{H^{s+\alpha-1}\left(\mathbb{R}^{n}\right)}\right) .
\end{aligned}
$$


Proof. The equation (18) can be written, in the variables $u$ and $u_{t}=v$, as the first order system,

$$
\left(\begin{array}{l}
u \\
v
\end{array}\right)_{t}=M\left(\begin{array}{l}
u \\
v
\end{array}\right), \quad M=\left(\begin{array}{ll}
0 & I \\
\Delta & 0
\end{array}\right)
$$

Then the semigroup $S(t)$ associated with the problem (19) is given by

$$
S(t)=\left(\begin{array}{cc}
\mathcal{F}^{-1} & 0 \\
0 & \mathcal{F}^{-1}
\end{array}\right)\left(\begin{array}{cc}
\cos (|\xi| t) & \frac{\sin (|\xi| t)}{|\xi|} \\
-|\xi| \sin (|\xi| t) & \cos (|\xi| t)
\end{array}\right)\left(\begin{array}{cc}
\mathcal{F} & 0 \\
0 & \mathcal{F}
\end{array}\right)
$$

So that, the proof follows as in Theorem 3 with $a=b$ and $\rho \equiv 1$.

In a similar way we have the coming corollary.

Corollary 3. Suppose $n \geq 2, s \in \mathbb{R}, 2<p<\infty$, $\frac{1}{p}+\frac{1}{p^{\prime}}=1$ and $\gamma=\frac{n+1}{2}\left(\frac{1}{p^{\prime}}-\frac{1}{p}\right)$. If $\Phi_{0} \in \dot{H}^{s+\alpha, p^{\prime}}\left(\mathbb{R}^{n}\right)$, $w_{0} \in \dot{H}^{s+\alpha-1, p^{\prime}}\left(\mathbb{R}^{n}\right)$ and $\Phi$ is a solution to the initial value problem in $\mathbb{R}^{n}$,

$$
\begin{gathered}
\Phi_{t t}-\Delta \Phi=0 \\
\Phi(x, 0)=\Phi_{0}(x), \quad \Phi_{t}(x, 0)=w_{0}(x) .
\end{gathered}
$$

Then the following estimate holds

$$
\begin{aligned}
& \|\Phi\|_{\dot{H}^{s, p}\left(\mathbb{R}^{n}\right)}+\left\|\Phi_{t}\right\|_{\dot{H}^{s-1, p}\left(\mathbb{R}^{n}\right)} \\
& \leq C|t|^{\frac{-(n-1)}{2}\left(\frac{1}{p^{\prime}}-\frac{1}{p}\right)}\left(\left\|\Phi_{0}\right\|_{\dot{H}^{s+\gamma, p^{\prime}}\left(\mathbb{R}^{n}\right)}+\left\|w_{0}\right\|_{\dot{H}^{s+\gamma-1, p^{\prime}}\left(\mathbb{R}^{n}\right)}\right) \text {. }
\end{aligned}
$$

\subsection{A Boussinesq-Benney-Luke system}

J. Quintero and A. Montes in [13] (see also [10]) reduced the study of the evolution of long water waves with small amplitude to studying solutions $(\Phi(x, y, t)), \eta(x, y, t)$ of the 2D-Boussinesq-Benney-Luke system,

$$
\left\{\begin{array}{r}
\left.\left(I-\frac{\mu}{2} \Delta\right) \eta_{t}+\Delta \Phi-\frac{2 \mu}{3} \Delta^{2} \Phi+\varepsilon \nabla \cdot(\eta \nabla \Phi)\right)=0 \\
\left(I-\frac{\mu}{2} \Delta\right) \Phi_{t}+\eta-\mu \sigma \Delta \eta+\frac{\varepsilon}{2}|\nabla \Phi|^{2}=0
\end{array}\right.
$$

where $\varepsilon$ is the amplitude parameter, $\mu$ is the long-wave parameter and $\sigma$ is the Bond number (associated with the surface tension). The variable $\Phi$ is the rescale nondimensional velocity potential on the bottom $z=0$, and the variable $\eta$ is the rescaled free surface elevation.

We will show how Corollary 1 and Theorem 2 are used to obtain Strichartz type estimates for the Boussinesq system. We first note that operators $A=I-\frac{\mu}{2} \Delta, B=I-\frac{2 \mu}{3} \Delta$ and $D=I-\mu \sigma \Delta$ are defined via the Fourier transform as

$$
\widehat{A}=1+\frac{\mu}{2}|\xi|^{2}, \widehat{B}=1+\frac{2 \mu}{3}|\xi|^{2}, \widehat{D}=1+\mu \sigma|\xi|^{2} .
$$

Using this, we can written the Boussinesq system as

$$
\left(\begin{array}{c}
\eta \\
\Phi
\end{array}\right)_{t}+M\left(\begin{array}{c}
\eta \\
\Phi
\end{array}\right)+G\left(\begin{array}{c}
\eta \\
\Phi
\end{array}\right)=0
$$

where $M$ is a linear operator and $G$ corresponds to the nonlinear part,

$$
\begin{gathered}
M=\left(\begin{array}{cc}
0 & \Delta A^{-1} B \\
A^{-1} D & 0
\end{array}\right) \\
G\left(\begin{array}{c}
\eta \\
\Phi
\end{array}\right)=\varepsilon\left(\begin{array}{c}
A^{-1}(\nabla \eta \cdot \nabla \Phi+\eta \Delta \Phi) \\
\frac{1}{2} A^{-1}\left(|\nabla \Phi|^{2}\right)
\end{array}\right) .
\end{gathered}
$$

and

Moreover, linear operator $M$ is the infinitesimal generator of a $C_{0}$-semigroup $S(t)$ defined as

$$
S(t)=\left(\begin{array}{cc}
\mathcal{F}^{-1} & 0 \\
0 & \mathcal{F}^{-1}
\end{array}\right) \widehat{e^{t M}}\left(\begin{array}{cc}
\mathcal{F} & 0 \\
0 & \mathcal{F}
\end{array}\right)
$$

where we have that

$$
\widehat{e^{t M}}=\left(\begin{array}{cc}
\cos (|\xi| \rho(|\xi|) t)|\xi| \varphi(\xi) \sin (|\xi| \rho(|\xi|) t) \\
-\frac{\sin (|\xi| \rho(|\xi|) t)}{|\xi| \varphi(\xi)} & \cos (|\xi| \rho(|\xi|) t)
\end{array}\right)
$$

where the functions $\varphi$ and $\rho$ are given by

$$
\varphi(\xi)=\sqrt{\frac{1+\beta|\xi|^{2}}{1+v|\xi|^{2}}}, \quad \rho(r)=\frac{\sqrt{\left(1+\beta r^{2}\right)\left(1+v r^{2}\right)}}{1+\delta r^{2}},
$$

with $\delta=\frac{\mu}{2}, \quad \beta=\frac{2 \mu}{3}$ and $\quad v=\mu \sigma$.

Lemma 9. $\rho$ satisfies the condition $[\mathbf{H}]$.

Proof. If $\rho_{j}(r)=\rho\left(2^{j} r\right)$, then we have that

$$
\begin{aligned}
h_{j}(r) & =\rho_{j}(r)+r \rho_{j}^{\prime}(r) \\
& =\frac{\sqrt{\left(1+\beta 4^{j} r^{2}\right)\left(1+v 4^{j} r^{2}\right)}}{1+\delta 4^{j} r^{2}} \\
& +\frac{\beta 4^{j} r^{2}}{1+\delta 4^{j} r^{2}} \sqrt{\frac{1+v 4^{j} r^{2}}{1+\beta 4^{j} r^{2}}} \\
& +\frac{v 4^{j} r^{2}}{1+\delta 4^{j} r^{2}} \sqrt{\frac{1+\beta 4^{j} r^{2}}{1+v 4^{j} r^{2}}} \\
& -\frac{2 \delta 4^{j} r^{2} \sqrt{\left(1+\beta 4^{j} r^{2}\right)\left(1+v 4^{j} r^{2}\right)}}{\left(1+\delta 4^{j} r^{2}\right)^{2}} \\
& =\frac{1+\delta 4^{j} r^{2}+2(\beta-\delta) 4^{j} r^{2}}{\left(1+\delta 4^{j} r^{2}\right)^{2} \sqrt{\left(1+\beta 4^{j} r^{2}\right)\left(1+v 4^{j} r^{2}\right)}} \\
& +\frac{2 v 4^{j} r^{2}+3 \beta v 4^{2 j} r^{4}+\delta \beta v 4^{3 j} r^{6}}{\left(1+\delta 4^{j} r^{2}\right)^{2} \sqrt{\left(1+\beta 4^{j} r^{2}\right)\left(1+v 4^{j} r^{2}\right)}} .
\end{aligned}
$$


Now, let $I=\left[r_{1}, r_{2}\right] \subset \mathbb{R}^{+}$. Using that $\beta>\delta>0$ and $v>0$, we see that for any $(r, j) \in I \times \mathbb{Z}$ holds the estimate

$$
\begin{aligned}
& h_{j}(r) \geq \\
& \frac{1+\delta 4^{j} r_{1}^{2}+2(\beta-\delta) 4^{j} r_{1}^{2}}{\left(1+\delta 4^{j} r_{2}^{2}\right)^{2} \sqrt{\left(1+\beta 4^{j} r_{2}^{2}\right)\left(1+v 4^{j} r_{2}^{2}\right)}} \\
& +\frac{2 v 4^{j} r_{1}^{2}+3 \beta v 4^{2 j} r_{1}^{4}+\delta \beta v 4^{3 j} r_{1}^{6}}{\left(1+\delta 4^{j} r_{2}^{2}\right)^{2} \sqrt{\left(1+\beta 4^{j} r_{2}^{2}\right)\left(1+v 4^{j} r_{2}^{2}\right)}}:=\pi_{j} .
\end{aligned}
$$

Note that

$$
\lim _{j \rightarrow-\infty} \pi_{j}=1, \quad \lim _{j \rightarrow \infty} \pi_{j}=\frac{\sqrt{\beta v}}{\delta}\left(\frac{r_{1}}{r_{2}}\right)^{6} .
$$

Hence, there exist $c_{0}>0$ such that for all $(r, j) \in I \times \mathbb{Z}$,

$$
\left|h_{j}(r)\right| \geq c_{0}
$$

On the other hand, a calculation shows that

$$
\begin{aligned}
& h_{j}^{\prime}(r) \\
& =\frac{2 \delta_{1} 4^{j} r+12 \beta v 4^{2 j} r^{3}+3 \delta \beta v 4^{3 j} r^{5}}{\left(1+\delta 4^{j} r^{2}\right)^{2} \sqrt{\left(1+\beta 4^{j} r^{2}\right)\left(1+v 4^{j} r^{2}\right)}} \\
& -\frac{4 \delta 4^{j} r\left(1+\delta_{1} 4^{j} r^{2}+3 \beta v 4^{2 j} r^{4}+\delta \beta v 4^{3 j} r^{6}\right)}{\left(1+\delta 4^{j} r^{2}\right)^{3} \sqrt{\left(1+\beta 4^{j} r^{2}\right)\left(1+v 4^{j} r^{2}\right)}} \\
& -\frac{4^{j} \beta r\left(1+\delta_{1} 4^{j} r^{2}+3 \beta v 4^{2 j} r^{4}+\delta \beta v 4^{3 j} r^{6}\right)}{\left(1+\delta 4^{j} r^{2}\right)^{2}\left(1+\beta 4^{j} r^{2}\right) \sqrt{\left(1+\beta 4^{j} r^{2}\right)\left(1+v 4^{j} r^{2}\right)}} \\
& -\frac{v 4^{j} r\left(1+\delta_{1} 4^{j} r^{2}+3 \beta v 4^{2 j} r^{4}+\delta \beta v 4^{3 j} r^{6}\right)}{\left(1+\delta 4^{j} r^{2}\right)^{2}\left(1+v 4^{j} r^{2}\right) \sqrt{\left(1+\beta 4^{j} r^{2}\right)\left(1+v 4^{j} r^{2}\right)}}
\end{aligned}
$$

where $\delta_{1}=2(\beta+v)-\delta$. Then for any $(r, j) \in I \times \mathbb{Z}$ we have that

$$
\begin{aligned}
& \left|h_{j}^{\prime}(r)\right| \\
& \leq \frac{2 \delta_{1} 4^{j} r_{2}+12 \beta v 4^{2 j} r_{2}^{3}+3 \delta \beta v 4^{3 j} r_{2}^{5}}{\left(1+\delta 4^{j} r_{1}^{2}\right)^{2} \sqrt{\left(1+\beta 4^{j} r_{1}^{2}\right)\left(1+v 4^{j} r_{1}^{2}\right)}} \\
& +\frac{4 \delta 4^{j} r_{2}\left(1+\delta_{1} 4^{j} r_{2}^{2}+3 \beta v 4^{2 j} r_{2}^{4}+\delta \beta v 4^{3 j} r_{2}^{6}\right)}{\left(1+\delta 4^{j} r_{1}^{2}\right)^{3} \sqrt{\left(1+\beta 4^{j} r_{1}^{2}\right)\left(1+v 4^{j} r_{1}^{2}\right)}} \\
& +\frac{4^{j} \beta r\left(1+\delta_{1} 4^{j} r_{2}^{2}+3 \beta v 4^{2 j} r_{2}^{4}+\delta \beta v 4^{3 j} r_{2}^{6}\right)}{\left(1+\delta 4^{j} r_{1}^{2}\right)^{2}\left(1+\beta 4^{j} r_{1}^{2}\right) \sqrt{\left(1+\beta 4^{j} r_{1}^{2}\right)\left(1+v 4^{j} r_{1}^{2}\right)}} \\
& +\frac{v 4^{j} r_{2}\left(1+\delta_{1} 4^{j} r_{2}^{2}+3 \beta v 4^{2 j} r_{2}^{4}+\delta \beta v 4^{3 j} r_{2}^{6}\right)}{\left(1+\delta 4^{j} r_{1}^{2}\right)^{2}\left(1+v 4^{j} r_{1}^{2}\right) \sqrt{\left(1+\beta 4^{j} r_{1}^{2}\right)\left(1+v 4^{j} r_{1}^{2}\right)}} .
\end{aligned}
$$

Then we find that

$$
\lim _{j \rightarrow-\infty} h_{j}^{\prime}(r)=0, \quad \lim _{j \rightarrow \infty}\left|h_{j}^{\prime}(r)\right| \leq \frac{3 \sqrt{\beta v}}{\delta} \frac{r_{2}^{5}}{r_{1}^{6}} .
$$

So, we conclude that there exist $c_{1}>0$ such that $\left|h_{j}^{\prime}(r)\right| \leq c_{1}$ for all $(j, r) \in \mathbb{Z} \times I$. Hence, for $c_{2}=c_{1} / c_{0}$ we concluded that

$$
\frac{\left|h_{j}^{\prime}(r)\right|}{\left|h_{j}(r)\right|} \leq c_{2}
$$

Next we will state the main results of this section.

Theorem 5. Suppose $s \in \mathbb{R}, 2 \leq p<\infty, \frac{1}{q}=\frac{1}{4}-\frac{1}{2 p}$ and $\alpha=\frac{3}{4}-\frac{3}{2 p}$. Then the semigroup $S(t)$ associated with system Boussinesq-Benney-Luke (20) satisfies the estimates

$\|S(t)(\eta, \Phi)\|_{L_{t}^{q}\left(\dot{H}^{s-1, p \times \dot{H}^{s, p}}\right)} \leq C\left(\|\eta\|_{\dot{H}^{s+\alpha-1}}+\|\Phi\|_{\dot{H}^{s+\alpha}}\right)$.

Proof. From previous lemma we have that $\pm \rho$ satisfies the condition $[\mathbf{H}]$. Now, note that $\varphi$ and $1 / \varphi$ are bounded functions and functions $\sin (|\xi| \rho(|\xi|) t)$ and $\cos (|\xi| \rho(|\xi|) t)$ are linear combinations of $e^{ \pm i|\xi| \rho(|\xi|) t}$. Then using the Corollary 1 for $n=2$, we have that

$$
\begin{aligned}
& \left\|\mathcal{F}^{-1}(\cos (|\xi| \rho(|\xi|) t) \widehat{\eta})\right\|_{L_{t}^{q}\left(\dot{H}^{s-1, p}\right)} \\
& +\left\|\mathcal{F}^{-1}(|\xi| \varphi(\xi) \sin (|\xi| \rho(|\xi|) t) \widehat{\Phi})\right\|_{L_{t}^{q}\left(\dot{H}^{s-1, p}\right)} \\
& \leq C\left(\|\eta\|_{\dot{H}^{s+\alpha-1}}+\|\Phi\|_{\dot{H}^{s+\alpha}}\right)
\end{aligned}
$$

and also that

$$
\begin{aligned}
& \left\|\mathcal{F}^{-1}\left(\frac{\sin (|\xi| \rho(|\xi|) t)}{|\xi| \varphi(\xi)} \widehat{\eta}\right)\right\|_{L_{t}^{q}\left(\dot{H}^{s, p}\right)} \\
& +\left\|\mathcal{F}^{-1}(\cos (|\xi| \rho(|\xi|) t) \widehat{\Phi})\right\|_{L_{t}^{q}\left(\dot{H}^{s, p}\right)} \\
& \leq C\left(\|\eta\|_{\dot{H}^{s+\alpha-1}}+\|\Phi\|_{\dot{H}^{s+\alpha}}\right) .
\end{aligned}
$$

Hence we obtain,

$\|S(t)(\eta, \Phi)\|_{L_{t}^{q}\left(\dot{H}^{s-1, p} \times \dot{H}^{s, p}\right)} \leq C\left(\|\eta\|_{\dot{H}^{s+\alpha-1}}+\|\Phi\|_{\dot{H}^{s+\alpha}}\right)$.

In a similar fashion we have the following theorem.

Theorem 6. Suppose $s \in \mathbb{R}, 2<p<\infty, \frac{1}{p}+\frac{1}{p^{\prime}}=1$ and $\gamma=\frac{3}{2}\left(\frac{1}{p^{\prime}}-\frac{1}{p}\right)$. Then there exist $C>0$ such that

$$
\begin{aligned}
& \|S(t)(\eta, \Phi)\|_{\dot{H}^{s-1, p} \times \dot{H}^{s, p}} \\
& \quad \leq C|t|^{\frac{-1}{2}\left(\frac{1}{p^{\prime}}-\frac{1}{p}\right)}\left(\|\eta\|_{\dot{H}^{s+\gamma-1, p^{\prime}}}+\|\Phi\|_{\dot{H}^{s+\gamma, p^{\prime}}}\right) .
\end{aligned}
$$

\subsection{A 2D Boussinesq Type System}

In this section we show Strichartz type estimates for solutions of associated linear problem with the Boussinesq type system in $\mathbb{R}^{1+2}$

$$
\left\{\begin{array}{l}
\eta_{t}+\Delta \Phi-\frac{\mu}{6} \Delta^{2} \Phi+\varepsilon \nabla \cdot(\eta \nabla \Phi)=0 \\
\Phi_{t}+\eta-\mu\left(\sigma-\frac{1}{2}\right) \Delta \eta+\frac{\varepsilon}{2}|\nabla \Phi|^{2}=0 .
\end{array}\right.
$$


with $\varepsilon, \mu, \sigma>0$. J. Quintero reduced the study of the evolution of long water with small amplitude to studying solutions $(\Phi, \eta)$ of this $2 \mathrm{D}$-Boussinesq system (see [15]).

If $\lambda=\frac{\mu}{6}, \beta=\mu\left(\sigma-\frac{1}{2}\right)>0, u=\partial_{1} \Phi$ and $v=\partial_{2} \Phi$ then formally $\partial_{2} u=\partial_{1} v$ and the system (21) is transformed into a first order system in the variables $\eta, u, v$ of the form

$$
\left(\begin{array}{l}
\eta \\
u \\
v
\end{array}\right)_{t}+M\left(\begin{array}{l}
\eta \\
u \\
v
\end{array}\right)+G\left(\begin{array}{l}
\eta \\
u \\
v
\end{array}\right)=0
$$

where

$$
M=\left(\begin{array}{ccc}
0 & A \partial_{1} & A \partial_{2} \\
B \partial_{1} & 0 & 0 \\
B \partial_{2} & 0 & 0
\end{array}\right)
$$

and $G$ is given by

$$
G\left(\begin{array}{l}
\eta \\
u \\
v
\end{array}\right)=-\varepsilon\left(\begin{array}{c}
\partial_{1}(\eta u)+\partial_{2}(u v) \\
u \partial_{1} u+v \partial_{1} v \\
u \partial_{2} u+v \partial_{2} v
\end{array}\right),
$$

with $A=I-\lambda \Delta$ and $B=I-\beta \Delta$. The linear operator $M$ is the infinitesimal generator of a $C_{0}-$ semigroup $S(t)$ given by

$$
S(t)=\left(\begin{array}{ccc}
\mathcal{F}^{-1} & 0 & 0 \\
0 & \mathcal{F}^{-1} & 0 \\
0 & 0 & \mathcal{F}^{-1}
\end{array}\right) \widehat{e^{t M}}\left(\begin{array}{lll}
\mathcal{F} & 0 & 0 \\
0 & \mathcal{F} & 0 \\
0 & 0 & \mathcal{F}
\end{array}\right),
$$

where $\widehat{e^{t M}}$ is defined as the matrix

$$
\left(\begin{array}{ccc}
C(\xi) & -i \varphi_{1}(\xi) S(\xi) & -i \varphi_{2}(\xi) S(\xi) \\
-i \zeta_{1}(\xi) S(\xi) & \phi_{1}(\xi) C(\xi) & \psi(\xi) C(\xi) \\
-i \zeta_{2}(\xi) S(\xi) \psi(\xi) \cos (|\xi| \rho(|\xi|) t) & \phi_{2}(\xi) C(\xi)
\end{array}\right)
$$

whit

$$
S(\xi)=\sin (|\xi| \rho(|\xi|) t), C(\xi)=\cos (|\xi| \rho(|\xi|) t)
$$

and the functions $\varphi_{k}, \zeta_{k}, \phi_{k}, \psi(k=1,2)$ are defined for $\xi=\left(\xi_{1}, \xi_{2}\right)$ by

$$
\begin{gathered}
\varphi_{k}(\xi)=\frac{\xi_{k}}{|\xi|} \sqrt{\frac{1+\lambda|\xi|^{2}}{1+\beta|\xi|^{2}}}, \quad \zeta_{k}(\xi)=\frac{\xi_{k}}{|\xi|} \sqrt{\frac{1+\beta|\xi|^{2}}{1+\lambda|\xi|^{2}}}, \\
\phi_{k}(\xi)=\frac{\xi_{k}^{2}}{|\xi|^{2}}, \quad \psi(\xi)=\frac{\xi_{1} \xi_{2}}{|\xi|^{2}}
\end{gathered}
$$

and $\rho$ is given by

$$
\rho(r)=\sqrt{\left(1+\lambda r^{2}\right)\left(1+\beta r^{2}\right)} .
$$

We notice that for $k=1,2$,

$$
\varphi_{k}, \zeta_{k}, \phi_{k}, \psi \in L^{\infty}\left(\mathbb{R}^{2}\right)
$$

Moreover, $\rho$ satisfies the condition $[\mathbf{H}]$. In fact,

$$
\begin{aligned}
h_{j}(r) & =\rho_{j}(r)+r \rho_{j}^{\prime}(r) \\
& =\sqrt{\left(1+\lambda 4^{j} r^{2}\right)\left(1+\beta 4^{j} r^{2}\right)} \\
& +\frac{(\lambda+\beta) 4^{j} r^{2}+2 \lambda \beta 4^{2 j} r^{4}}{\sqrt{\left(1+\lambda 4^{j} r^{2}\right)\left(1+\beta 4^{j} r^{2}\right)}} \\
& =\frac{1+2(\lambda+\beta) 4^{j} r^{2}+3 \lambda \beta 4^{2 j} r^{4}}{\sqrt{\left(1+\lambda 4^{j} r^{2}\right)\left(1+\beta 4^{j} r^{2}\right)}} .
\end{aligned}
$$

Then it is easy to see that for any interval $I=\left[r_{1}, r_{2}\right] \subset \mathbb{R}^{+}$, there exist $c_{1}>0$ such that for all $(j, r) \in \mathbb{Z} \times I$,

$$
\left|h_{j}(r)\right| \geq c_{1} .
$$

Now, we see that

$$
\begin{aligned}
h_{j}^{\prime}(r) & =\frac{3(\lambda+\beta) 4^{j} r+2(\lambda+\beta)^{2} 4^{2 j} r^{3}+10 \lambda \beta 4^{2 j} r^{3}}{\left(1+\lambda 4^{j} r^{2}\right)\left(1+\beta 4^{j} r^{2}\right) \sqrt{\left(1+\lambda 4^{j} r^{2}\right)\left(1+\beta 4^{j} r^{2}\right)}} \\
& +\frac{9 \lambda \beta(\lambda+\beta) 4^{3 j} r^{5}+6(\lambda \beta)^{2} 4^{4 j} r^{7}}{\left(1+\lambda 4^{j} r^{2}\right)\left(1+\beta 4^{j} r^{2}\right) \sqrt{\left(1+\lambda 4^{j} r^{2}\right)\left(1+\beta 4^{j} r^{2}\right)}} .
\end{aligned}
$$

So that,

$$
\begin{aligned}
& \frac{\left|h_{j}^{\prime}(r)\right|}{\left|h_{j}(r)\right|}= \\
& \frac{3(\lambda+\beta) 4^{j} r+2(\lambda+\beta)^{2} 4^{2 j} r^{3}+10 \lambda \beta 4^{2 j} r^{3}}{\left(1+\lambda 4^{j} r^{2}\right)\left(1+\beta 4^{j} r^{2}\right)\left(1+2(\lambda+\beta) 4^{j} r^{2}+3 \lambda \beta 4^{2 j} r^{4}\right)} \\
& +\frac{9 \lambda \beta(\lambda+\beta) 4^{3 j} r^{5}+6(\lambda \beta)^{2} 4^{4 j} r^{7}}{\left(1+\lambda 4^{j} r^{2}\right)\left(1+\beta 4^{j} r^{2}\right)\left(1+2(\lambda+\beta) 4^{j} r^{2}+3 \lambda \beta 4^{2 j} r^{4}\right)} .
\end{aligned}
$$

Then a simple calculation shows that there exist $c_{2}>0$ such that

$$
\frac{\left|h_{j}^{\prime}(r)\right|}{\left|h_{j}(r)\right|^{2}} \leq c_{2} \text {. }
$$

In a similar fashion as for the previous models we have the following theorems. We use the notation

$$
\dot{Y}^{s, p}=\dot{H}^{s, p} \times \dot{H}^{s, p} \times \dot{H}^{s, p} .
$$

Theorem 7. Suppose $s \in \mathbb{R}, 2 \leq p<\infty, \frac{1}{q}=\frac{1}{4}-\frac{1}{2 p}$ and $\alpha=\frac{3}{4}-\frac{3}{2 p}$. Then the semigroup $S(t)$ satisfies the estimates

$$
\begin{aligned}
& \left.\|S(t)(\eta, u, v)\|_{L_{t}^{q}(\dot{Y} s, p}\right) \\
& \leq C\left(\|\eta\|_{\dot{H}^{s+\alpha}}+\|u\|_{\dot{H}^{s+\alpha}}+\|v\|_{\dot{H}^{s+\alpha}}\right) .
\end{aligned}
$$

Theorem 8. Suppose $s \in \mathbb{R}, 2<p<\infty, \frac{1}{p}+\frac{1}{p^{\prime}}=1$ and $\gamma=$ $\frac{3}{2}\left(\frac{1}{p^{\prime}}-\frac{1}{p}\right)$. Then the semigroup $S(t)$ satisfies the estimates

$$
\begin{aligned}
& \|S(t)(\eta, u, v)\|_{\dot{Y}^{s, p},} \\
& \leq C|t|^{\frac{-1}{2}\left(\frac{1}{p^{\prime}}-\frac{1}{p}\right)}\left(\|\eta\|_{\dot{H}^{s+\gamma, p^{\prime}}}+\|u\|_{\dot{H}^{s+\gamma, p^{\prime}}}+\|v\|_{\dot{H}^{s+\gamma, p^{\prime}}}\right) .
\end{aligned}
$$




\section{Asymptotic behavior of solutions in the energy space of the (gBL)}

In present section, for $m \geq 4$ and the initial data small and belongs to a suitable Sobolev space, we study the asymptotic behavior of global solutions in the energy space of the (gBL) equation as $t \rightarrow \pm \infty$, Throughout this section we use the following result concerning the global well-posedness for the generalized Benney-Luke equation in the energy space $V^{2} \times H^{1}$ (see J. Quintero [17]). For this particular problem, it is known that there exists a energy functional $e(t)=\mathcal{E}\left(\Phi, \Phi_{t}\right)(t)$ which is conserved in time on solutions $\Phi$ for the generalized Benney-Luke equation. The energy $\mathcal{E}$ is given by

$\mathcal{E}(\Phi, w)=\frac{1}{2} \int_{\mathbb{R}^{2}}\left(|\nabla \Phi|^{2}+a|\Delta \Phi|^{2}+|w|^{2}+b|\nabla w|^{2}\right) d x d y$.

We note that there exist $C=C(a, b)$ such that for all $(\Phi, w) \in \mathcal{V}^{2} \times H^{1}$

$$
C^{-1}\|(\Phi, w)\|_{\mathcal{V}^{2} \times H^{1}}^{2} \leq \mathcal{E}(\Phi, w) \leq C\|(\Phi, w)\|_{\mathcal{V}^{2} \times H^{1}}^{2} .
$$

Theorem 9. ([17]) Let $m \geq 1$ and $\left(\Phi_{0}, w_{0}\right) \in \mathcal{V}^{2} \times H^{1}$. Then the Cauchy problem associated with the $(g B L)$ equation has an unique global solution $\Phi$ with

$$
\Phi \in C\left((-\infty, \infty): \mathcal{V}^{2}\right) \cap C^{1}\left((-\infty, \infty): H^{1}\right),
$$

that satisfies the initial condition

$$
\Phi(0, \cdot)=\Phi_{0}, \quad \Phi_{t}(0, \cdot)=w_{0} .
$$

Moreover, $\mathcal{E}\left(\Phi, \Phi_{t}\right)(t)=\mathcal{E}\left(\Phi_{0}, w_{0}\right)$ for $t \in \mathbb{R}$ and we also have that the following estimate holds

$$
\left\|\left(\Phi(t), \Phi_{t}(t)\right)\right\|_{\mathcal{V}^{2} \times H^{1}} \leq C\left\|\left(\Phi_{0}, w_{0}\right)\right\|_{\mathcal{V}^{2} \times H^{1}} .
$$

In order to obtain the result which describe the asymptotic behavior of the solutions in the energy space of the ( $\mathrm{gBL}$ ) equation we will use the following lemma.

Lemma 10. Let $2<p<\infty, \frac{1}{p^{\prime}}+\frac{1}{p}=1$ and $\gamma=\frac{3}{2}\left(\frac{1}{p^{\prime}}-\frac{1}{p}\right)$. Then there exist $C>0$ such that

$$
\begin{aligned}
& (1+|t|)^{\frac{1}{2}\left(\frac{1}{p^{\prime}}-\frac{1}{p}\right)}\|S(t)(\Phi, w)\|_{\dot{H}^{1, p} \times L^{p}} \\
& \quad \leq C\left(\|(\Phi, w)\|_{\dot{H}^{1+\gamma, p^{\prime}} \times \dot{H}^{\gamma, p^{\prime}}}+\|(\Phi, w)\|_{\mathcal{V}^{2} \times H^{1}}\right) .
\end{aligned}
$$

Proof. First we note that if $(\Phi, w) \in \mathcal{V}^{2} \times H^{1}$ then we have that

$$
\|w\|_{H^{1}}=\|w\|_{L^{2}}+\|w\|_{\dot{H}^{1}}, \quad\|\Phi\|_{\mathcal{V}^{2}}=\|\Phi\|_{\dot{H}^{1}}+\|\Phi\|_{\dot{H}^{2}} .
$$

Therefore

$$
\|(\Phi, w)\|_{\mathcal{V}^{2} \times H^{1}}=\|\Phi\|_{\dot{H}^{1}}+\|\Phi\|_{\dot{H}^{2}}+\|w\|_{L^{2}}+\|w\|_{\dot{H}^{1}} .
$$

Now, if $(\Phi, w) \in \mathcal{V}^{2} \times H^{1}$ then using Theorem 3 with $p=$ 2, $q=\infty, \alpha=0$ and $s=1,2$ we have that there is $C>0$ such that for all $t \in \mathbb{R}$,

$$
\|S(t)(\Phi, w)\|_{\dot{H}^{1} \times L^{2}} \leq C\|(\Phi, w)\|_{\dot{H}^{1} \times L^{2}},
$$

and

$$
\|S(t)(\Phi, w)\|_{\dot{H}^{2} \times \dot{H}^{1}} \leq C\|(\Phi, w)\|_{\dot{H}^{2} \times H^{1}}
$$

Then we find that

$$
\|S(t)(\Phi, w)\|_{\mathcal{V}^{2} \times H^{1}} \leq C\|(\Phi, w)\|_{\mathcal{V}^{2} \times H^{1}} .
$$

Thus, since $L^{p} \hookrightarrow H^{1}$ we have that

$$
\begin{aligned}
\|S(t)(\Phi, w)\|_{\dot{H}^{1, p} \times L^{p}} & \leq C\|S(t)(\Phi, w)\|_{\mathcal{V}^{2} \times H^{1}} \\
& \leq C\|(\Phi, w)\|_{\mathcal{V}^{2} \times H^{1}} .
\end{aligned}
$$

On the other hand, by Theorem 4 we see that

$$
\begin{aligned}
|t|^{\frac{1}{2}\left(\frac{1}{p^{\prime}}-\frac{1}{p}\right)}\|S(t)(\Phi, w)\|_{\dot{H}^{1, p} \times L^{p}} & \leq C\|(\Phi, w)\|_{\dot{H}^{1+\gamma, p^{\prime}} \times \dot{H}^{\gamma}, p^{\prime}} .
\end{aligned}
$$

Hence, combining the previous inequalities yields the result. $\square$

We will need the following result.

Lemma 11. ([19], [22]) If $r_{1}, r_{2}>0$ and $\max \left\{r_{1}, r_{2}\right\}>1$, then

$\int_{-\infty}^{\infty}(1+|t-\tau|)^{-r_{1}}(1+|\tau|)^{-r_{2}} d \tau \leq C(1+|t|)^{-\min \left\{r_{1}, r_{2}\right\}}$.

Theorem 10. Let fixed $m \geq 4$. Suppose $2 m<p<\infty$, $\frac{1}{p^{\prime}}+\frac{1}{p}=1$ and $\gamma=\frac{3}{2}\left(\frac{1}{p^{\prime}}-\frac{1}{p}\right)$. Then there exist $\delta>0$ and $R=R(\delta)>0$ such that if the initial condition $\left(\Phi_{0}, w_{0}\right) \in \dot{H}^{1+\gamma, p^{\prime}} \times \dot{H}^{\gamma, p^{\prime}} \cap \mathcal{V}^{2} \times H^{1}$ with

$$
\left\|\left(\Phi_{0}, w_{0}\right)\right\|_{\dot{H}^{1+\gamma, p^{\prime} \times \dot{H}^{\gamma, p^{\prime}}}}+\left\|\left(\Phi_{0}, w_{0}\right)\right\|_{\mathcal{V}^{2} \times H^{1}}<\delta,
$$

then the corresponding solution of Cauchy problem associated with the $(\mathrm{gBL})$ equation provided by Theorem (9) satisfies

$$
\sup _{t \in \mathbb{R}}(1+|t|)^{\frac{1}{2}\left(\frac{1}{p^{\prime}}-\frac{1}{p}\right)}\left\|\left(\Phi(t), \Phi_{t}(t)\right)\right\|_{\dot{H}^{1, p} \times L^{p}} \leq R .
$$

Proof. Note that the smallness condition (25) promptly implies the existence global solutions in the space $V^{2} \times H^{1}$. Without loss of generality we restrict ourselves to the case $t>0$. Recall that if $w=\Phi_{t}$ then the (gBL) equation can be written as the system (17). In which case, the solution of Cauchy problem with initial condition $\left(\Phi_{0}, w_{0}\right)$ is given by the expression

$$
\begin{aligned}
(\Phi(t), w(t)) & =S(t)\left(\Phi_{0}, w_{0}\right) \\
& -\int_{0}^{t} S(t-\tau) G(\Phi(\tau), w(\tau)) d \tau .
\end{aligned}
$$


Let $\beta=\frac{1}{2}\left(\frac{1}{p^{\prime}}-\frac{1}{p}\right)$, then using Lemma 10 we find that

$$
\begin{aligned}
& \|(\Phi, w)\|_{\dot{H}^{1, p} \times L^{p}} \\
& \leq C \delta(1+t)^{-\beta} \\
& +\int_{0}^{t}(1+t-\tau)^{-\beta} \times \\
& \times\left(\|G(\Phi, w)\|_{\dot{H}^{1+\gamma, p^{\prime} \times H^{\gamma, p^{\prime}}}}+\|G(\Phi, w)\|_{\mathcal{V}^{2} \times H^{1}}\right) d \tau .
\end{aligned}
$$

Now, using Lemma 1 and definition of $B$ we see that

$$
\begin{aligned}
& \|G(\Phi, w)\|_{\dot{H}^{1+\gamma, p^{\prime} \times \dot{H}^{\gamma, p^{\prime}}}}+\|G(\Phi, w)\|_{\mathcal{V}^{2} \times H^{1}} \\
& =\left\|B^{-1} F\left(w, \Phi_{x}, \Phi_{y}\right)\right\|_{\dot{H}^{\gamma, p^{\prime}}}+\left\|B^{-1} F\left(w, \Phi_{x}, \Phi_{y}\right)\right\|_{H^{1}} \\
& \leq 2 C\left\|B^{-1} F\left(w, \Phi_{x} \Phi_{y}\right)\right\|_{H^{2, p^{\prime}}} \\
& \leq C\left\|F\left(w, \Phi_{x}, \Phi_{y}\right)\right\|_{L^{p^{\prime}}} \text {. }
\end{aligned}
$$

Recall that

$$
\begin{aligned}
F\left(w, \Phi_{x} \Phi_{y}\right) & =m w\left(\left(\Phi_{x}\right)^{m-1} \Phi_{x x}\right. \\
& \left.+\left(\Phi_{y}\right)^{m-1} \Phi_{y y}\right)+2\left(\left(\Phi_{x}\right)^{m} w_{x}+\left(\Phi_{y}\right)^{m} w_{y}\right) .
\end{aligned}
$$

To illustrate the type computation we consider the terms $w\left(\Phi_{x}\right)^{m-1} \Phi_{x x}$ and $\left(\Phi_{x}\right)^{m} w_{x}$. In fact, if $r=\frac{2 p}{p-2 m}>2$, by Hölder inequality we have that

$$
\begin{aligned}
\left\|w\left(\Phi_{x}\right)^{m-1} \Phi_{x x}\right\|_{L^{p^{\prime}}} & \leq\left\|\Phi_{x}\right\|_{L^{p}}^{m-1}\left\|\Phi_{x x}\right\|_{L^{2}}\|w\|_{L^{r}} \\
& \leq C\|\Phi\|_{\dot{H}^{1, p}}^{m-1}\|\Phi\|_{\mathcal{V}^{2}}\|w\|_{H^{1}} \\
& \leq C\|(\Phi, w)\|_{\dot{H}^{1, p} \times L^{p}}^{m-1}\|(\Phi, w)\|_{\mathcal{V}^{2} \times H^{1}}^{2} .
\end{aligned}
$$

Also we have that

$$
\begin{aligned}
\left\|\left(\Phi_{x}\right)^{m} w_{x}\right\|_{L^{p^{\prime}}} & \leq\left\|\Phi_{x}\right\|_{L^{p}}^{m-1}\left\|w_{x}\right\|_{L^{2}}\left\|\Phi_{x}\right\|_{L^{r}} \\
& \leq C\|\Phi\|_{\dot{H}^{1, p}}^{m-1}\|w\|_{H^{1}}\|\Phi\|_{\mathcal{V}^{2}} \\
& \leq C\|(\Phi, w)\|_{\dot{H}^{1, p} \times L^{p}}^{m-1}\|(\Phi, w)\|_{\mathcal{V}^{2} \times H^{1}}^{2}
\end{aligned}
$$

In a similar way we have the same type of estimate for the other terms. Now, if for $T>0$ we introduce the notation

$$
K(T)=\sup _{t \in[0, T]}(1+t)^{\beta}\|(\Phi(t), w(t))\|_{\dot{H}^{1, p} \times L^{p}},
$$

and using inequality (24) follows that

$$
\begin{aligned}
& \|(\Phi, w)\|_{\dot{H}_{p}^{1} \times L_{p}} \\
& \leq C \delta(1+t)^{-\beta} \\
& +C \int_{0}^{t}(1+t-\tau)^{-\beta} \times \\
& \times\left(\|(\Phi(\tau), w(\tau))\|_{\dot{H}^{1, p} \times L^{p}}^{m-1}\|(\Phi(\tau), w(\tau))\|_{\mathcal{V}^{2} \times H^{1}}^{2}\right) d \tau \\
& \leq C \delta(1+t)^{-\beta} \\
& +C \delta^{2} K(T)^{m-1} \int_{0}^{t}(1+t-\tau)^{-\beta}(1+\tau)^{-\beta(m-1)} d \tau .
\end{aligned}
$$

Then, multiplying the previous inequality by $(1+t)^{\beta}$ and using Lemma 11 we have that

$$
\begin{aligned}
K(T) & \leq C \delta+C \delta^{2} K(T)^{m-1}(1+t)^{\beta} \times \\
& \times \int_{0}^{t}(1+t-\tau)^{-\beta}(1+\tau)^{-\beta(m-1)} d \tau \\
& \leq C \delta+C \delta^{2} K(T)^{m-1} .
\end{aligned}
$$

Note that the hypothesis $m \geq 4$ guarantees that

$$
m^{2}-4 m+1>0
$$

and since $p>2 m$ then we see that

$$
\beta(m-1)=\max \{\beta, \beta(m-1)\}>1 .
$$

Next, Consider the function $f_{\delta}(x)=C \delta+C \delta^{2} x^{m-1}-x$. Since $f_{\delta}(0)=C \delta$ and for any $x>0, f_{\delta}(x) \rightarrow-x$ as $\delta \rightarrow 0$ then there exist $\delta_{0}>0$ such that for $\delta \in\left(0, \delta_{0}\right), f_{\delta}$ has a positive zero. Let $R=R(\delta)$ be the first positive zero of this function. The estimates just obtained imply that $f_{\delta}(K(T)) \geq 0$ and so $K(T) \leq R$, since $f_{\delta}$ changes its monotonicity only once in $\mathbb{R}$.

Remark. From the condition (27) we see that it suffices to take $m>2+\sqrt{3}$ in Theorem 10 .

The decay in (26) allows us to study the asymptotic behavior of such global solutions in the energy space as $t \rightarrow \pm \infty$. We will show under the smallness condition (25) that for the couple $\left(\Phi(t), \Phi_{t}(t)\right)$ there are associated elements $\left(\Phi_{+}, w_{+}\right),\left(\Phi_{-}, w_{-}\right)$such that

$$
\left(\Phi(t), \Phi_{t}(t)\right) \sim S(t)\left(\Phi_{+}, w_{+}\right)
$$

and

$$
\left(\Phi(t), \Phi_{t}(t)\right) \sim S(t)\left(\Phi_{-}, w_{-}\right),
$$

as $t \rightarrow \infty$ and $t \rightarrow-\infty$ respectively. More precisely we have the following theorems.

Theorem 11. Let $\Phi(t)$ be the solution of the Cauchy problem associated with the $(g B L)$ equation introduced by Theorem 10. If $w=\Phi_{t}$, then there exist a unique $\left(\Phi_{+}, w_{+}\right) \in \mathcal{V}^{2} \times H^{1}$ such that

$$
\left\|(\Phi(t), w(t))-S(t)\left(\Phi_{+}, w_{+}\right)\right\|_{\mathcal{V}^{2} \times H^{1}} \rightarrow 0 \quad \text { as } \quad t \rightarrow \infty .
$$

Proof. The proof of this theorem is quite standard and it follows similar arguments used to other Boussinesq type models (see [5], [12]). Define

$$
\begin{aligned}
\left(\Phi_{+}, w_{+}\right) & =\left(\Phi_{0}, w_{0}\right) \\
& -\int_{0}^{\infty} S(-\tau) G(\Phi(\tau), w(\tau)) d \tau .
\end{aligned}
$$


Then we have that

$$
\begin{aligned}
& \left\|\int_{0}^{t} S(-\tau) G(\Phi(\tau), w(\tau)) d \tau\right\|_{\mathcal{V}^{2} \times H^{1}} \\
& \leq C \int_{0}^{t}\|G(\Phi(\tau), w(\tau))\|_{\mathcal{V}^{2} \times H^{1}} d \tau \\
& \leq C \int_{0}^{t}\left\|B^{-1} F\left(w, \Phi_{x}, \Phi_{y}\right)\right\|_{H^{1}} d \tau \\
& \leq C \int_{0}^{t}\left\|B^{-1} F\left(w, \Phi_{x}, \Phi_{y}\right)\right\|_{H^{2, p^{\prime}}} d \tau \\
& \leq C \int_{0}^{t}\|(\Phi(\tau), w(\tau))\|_{\dot{H}^{1, p} \times L^{p}}^{m-1}\|(\Phi(\tau), w(\tau))\|_{\mathcal{V}^{2} \times H^{1}} d \tau \\
& \leq C \int_{0}^{t}(1+\tau)^{-\frac{1}{2}\left(\frac{1}{p^{\prime}}-\frac{1}{p}\right)(m-1)} d \tau \leq \text { const, }
\end{aligned}
$$

which proves that the integral in (28) converges in $V^{2} \times$ $H^{1}$. A similar argument and the formula

$$
\begin{aligned}
S(-t)(\Phi(t), w(t)) & -\left(\Phi_{+}, w_{+}\right) \\
& =\int_{t}^{\infty} S(-\tau) G(\Phi(\tau), w(\tau)) d \tau
\end{aligned}
$$

show that

$$
\left\|S(-t)(\Phi(t), w(t))-\left(\Phi_{+}, w_{+}\right)\right\|_{\mathcal{V}^{2} \times H^{1}} \rightarrow 0 \text { as } t \rightarrow \infty .
$$

By (24) we see that $\|(\Phi(t), w(t))\|_{\mathcal{V}^{2} \times H^{1}}$ is bounded, so $S(-t)(\Phi(t), w(t))$ converges weakly in $V^{2} \times H^{1}$, and we concluded that as $t \rightarrow \infty$,

$$
\begin{aligned}
\| S(-t) & (\Phi(t), w(t))-\left(\Phi_{+}, w_{+}\right) \|_{\mathcal{V}^{2} \times H^{1}} \\
& =\left\|(\Phi(t), w(t))-S(t)\left(\Phi_{+}, w_{+}\right)\right\|_{\mathcal{V}^{2} \times H^{1}} \rightarrow 0 .
\end{aligned}
$$

Theorem 12. Let $\Phi(t)$ be the solution of the Cauchy problem associated with the $(g B L)$ equation introduced by Theorem 10. If $w=\Phi_{t}$, then there exist a unique $\left(\Phi_{-}, w_{-}\right) \in \mathcal{V}^{2} \times H^{1}$ such that

$\left\|(\Phi(t), w(t))-S(t)\left(\Phi_{-}, w_{-}\right)\right\|_{\mathcal{V}^{2} \times H^{1}} \rightarrow 0 \quad$ as $\quad t \rightarrow-\infty$.

Proof. The result is obtained by setting,

$$
\left(\Phi_{-}, w_{-}\right)=\left(\Phi_{0}, w_{0}\right)-\int_{-\infty}^{0} S(-\tau) G(\Phi(\tau), w(\tau)) d \tau
$$

\section{Acknowledgement}

J. Quintero was supported by the Mathematics Department at Universidad del Valle (Colombia) under the project C.I. 910. A. Montes is a graduate student at the Doctorate Math Program at Universidad del Valle under the financial support of Universidad del Cauca (Colombia).

\section{References}

[1] A. Biswas, Soliton Solutions of the Perturbed Resonant Nonlinear Schrdingers Equation with Full Nonlinearity by Semi-inverse Variational Principle, Quantum Physics Letters, 1, 79-83 (2012).

[2] M. Ablowitz , A. Fokas, Musslimani H. . On a new non-local formulation of water waves. Journal of Fluids Mechanics, 562, 313-341 (2006).

[3] D. J. Benney, J. C. Luke, Interactions of permanent waves of finite amplitude. J. Math. Phys, 43, 309-313 (1964).

[4] N. Taghizadeh, M. Mirzazadeh, M. Akbari, M. Rahimian, Exact Soliton Solutions for Generalized Equal Width Equation, Mathematical Sciences Letters, 2, 99-106 (2013).

[5] L. Farah, F. Linares, A. Pastor, A note on the 2D generalized Zakharov-Kuznetsov equation: Local, global, and scattering results, J. Diff. Eq., 253, 2558-2571 (2012).

[6] A. Kara, H. Triki, A. Biswas, Conservation laws of the Bretherton Equation. Appl. Math. Inf. Sci. 7, 877-879 (2013).

[7] Y. Kivshar, G. Agrawal, Optical Solitons: From Fibers to Photonic Crystals, Academic Press, 1 (Ed.), (2003).

[8] H. Lindblad, C. D. Sogge. On existence and scattering with minimal regularity for semilinear wave equations. J. Funct. Anal., 130, 357-426 (1995).

[9] P. A. Milewski and J. B. Keller, Three dimensional water waves. Studies Appl. Math., 37, 149-166 (1996).

[10] A. M. Montes. Boussinesq-Benney-Luke type systems related with water waves models, Doctoral Thesis, Universidad del Valle - Colombia, (2013).

[11] L. Paumond, Towards a rigorous derivation of the fifth order KP equation. Math. Comp. in Simulation, 69, 477-491 (2005).

[12] G. Ponce, L. Vega. Nonlinear small data scattering for the generalized Korteweg-de Vries equation, J. Funct. Anal., 90, 445-457 (1990).

[13] J. R. Quintero , A. M. Montes, Existence, physical sense and analyticity of solitons for a 2D Boussinesq-Benney-Luke System, submitted (2013).

[14] J. R. Quintero, R. L. Pego. Two-dimensional solitary waves for a Benney-Luke equation, Phisica D, 45, 476-496 (1999).

[15] J. R. Quintero. Solitary water waves for a 2D Boussinesq type system, J. Part. Diff. Eq., 23-3, 251-280 (2010).

[16] J. R. Quintero. Existence and analyticity of lump solutions for generalized Benney-Luke equations, Rev. Col. Mat., 36 , 71-95 (2002).

[17] J. R. Quintero. A Remark on the Cauchy Problem for the Generalized Benney-Luke Equation, Differential and Integral Equations, 21, 859-890 (2008).

[18] J. R. Quintero, Solitons and Periodic Travelling Waves for the 2D- Generalized Benney-Luke Equation, Journal of Applicable Analysis, 86-3, 331-351 (2007).

[19] M. Reed, Abstract non-linear wave equation, SpringerVerlag, 507 (1976)

[20] S. Selberg. Lecture Notes Math, 632, (2001). PDE.

[21] I. E. Segal, Space-time decay dor solutions of wave equations, Adv. in Math., 22, 304-311 (1976).

[22] I. E. Segal, Dispersion for nonlinear relativistic equation, II. Ann. Scuola. Norm Sup. ser 4, t. 1, 459-497 (1968).

[23] A. M. A. El-Sayed, H. H. G Hashem, Y. M. Y. Omar, Positive continuous solution of a quadratic integral equation of fractional orders, Mathematical Sciences Letters, 2, 19-27 (2013). 
[24] R. S. Strichartz, Restrictions of Fourier transforms to quadratic surfaces and decay of solutions of wave equations, Duke Math. J. 44, 705-714 (1977).

[25] T. Wolff. Lectures on Harmonic analysis, University Lectures Series, 29 (2003).

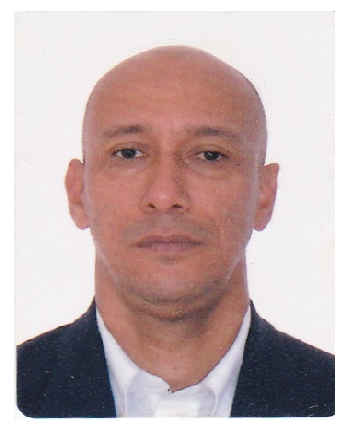

José R. Quintero is Titular Professor of Universidad del Valle (Cali-Colombia). He received the MS degree in mathematics from Universidad del Valle and the $\mathrm{Ph}$. D. degree from University of Maryland (College Park-USA). In the past years, J. Quintero has been interested in the derivation and the analysis of nonlinear dispersive water wave model.

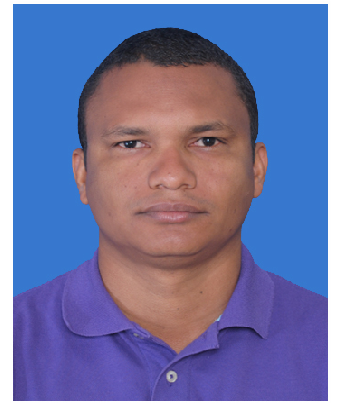

Alex M. Montes is Titular Professor of Universidad del Cauca (Popayán-Colombia).

He received the MS degree in mathematics from Universidad del Valle (Cali-Colombia). $\quad \mathrm{He}$ is expecting to finish his $\mathrm{Ph}$. D. studies at Universidad del Valle by August 2013. A.

Montes is interested in the derivation and the analysis of nonlinear dispersive water wave model. 